COMMUNICATIONS IN

ANALYSIS AND GEOMETRY

Volume 13, Number 3, 527-559, 2005

\title{
The equivariant cohomology of hypertoric varieties and their real loci
}

\author{
Megumi Harada and Tara S. Holm ${ }^{1}$
}

\begin{abstract}
Let $M$ be a Hamiltonian $T$ space with a proper moment map, bounded below in some component. In this setting, we give a combinatorial description of the $T$-equivariant cohomology of $M$, extending results of Goresky, Kottwitz and MacPherson and techniques of Tolman and Weitsman. Moreover, when $M$ is equipped with an antisymplectic involution $\sigma$ anticommuting with the action of $T$, we also extend to this non-compact setting the "mod 2" versions of these results to the real locus $Q:=M^{\sigma}$ of $M$. We give applications of these results to the theory of hypertoric varieties.
\end{abstract}

\section{Introduction.}

We present here two main results and demonstrate their use through several explicit computations. In the first result, we generalize to the non-compact setting a theorem of Goresky, Kottwitz, and MacPherson that computes $T=T^{n}$-equivariant cohomology rings of compact Hamiltonian $T$ spaces satisfying some technical conditions [8]. Suppose, in addition, that $M$ is equipped with an antisymplectic involution $\sigma$ that anticommutes with the $T$ action. In the second result, we generalize to the non-compact setting theorems $[4,5,7,18]$ that compute the equivariant cohomology of the real locus $Q:=M^{\sigma}$ of a Hamiltonian $T$ space $M$ satisfying similar technical conditions. The motivating examples for this paper are the hypertoric varieties studied in $[3,14,15,12,11]$ and their real loci. We present these in detail.

We first recall the basic idea of the theorem of Goresky, Kottwitz, and MacPherson, which we call the GKM theorem. For a compact Hamiltonian $T$ space $M$, Kirwan showed [13] that the inclusion $M^{T} \hookrightarrow M$ induces an injection $H_{T}^{*}(M ; \mathbb{Q}) \hookrightarrow H_{T}^{*}\left(M^{T} ; \mathbb{Q}\right)$ in equivariant cohomology. Since $T$ acts trivially on $M^{T}$, when $M^{T}$ consists of isolated points, the ring $H_{T}^{*}\left(M^{T} ; \mathbb{Q}\right)$

\footnotetext{
${ }^{1}$ The second author was supported by a National Science Foundation Postdoctoral Research Fellowship.
} 
is a direct product of polynomial rings

$$
H_{T}^{*}\left(M^{T} ; \mathbb{Q}\right) \cong \prod_{p \in M^{T}} H_{T}^{*}(p ; \mathbb{Q}) \cong \prod_{p \in M^{T}} \operatorname{Sym}\left(\mathfrak{t}^{*}\right) .
$$

Hence, in order to compute the equivariant cohomology $\operatorname{ring} H_{T}^{*}(M ; \mathbb{Q})$, it suffices to identify its image in $H_{T}^{*}\left(M^{T} ; \mathbb{Q}\right)$. Suppose, in addition, that the $T$-isotropy weights $\left\{\alpha_{p, i}\right\}$ are pairwise linearly independent at each fixed point $p$. The GKM theorem then asserts that the image of $H_{T}^{*}(M ; \mathbb{Q})$ in $H_{T}^{*}\left(M^{T} ; \mathbb{Q}\right)$ is the same as that of the one-skeleton of $M$, which in turn can be described combinatorially in terms of a graph $\Gamma$ and the $T$-isotropy data. Thus, the computation of $H_{T}^{*}(M ; \mathbb{Q})$ is translated into a problem of combinatorics.

In the setting of compact Hamiltonian $T$ manifolds equipped with an additional antisymplectic involution $\sigma$, we define the real locus $Q:=M^{\sigma}$ of $M$, which is equipped with an action of the discrete subgroup $T_{\mathbb{R}}:=\left(\mathbb{Z}_{2}\right)^{n}$ of $T$. The mod 2 GKM theorem $[4,18]$ gives a similar combinatorial description of the image of the equivariant cohomology of the real locus $Q:=M^{\sigma}$ as a subring of the equivariant cohomology (with $\mathbb{Z}_{2}$ coefficients) of $Q^{T_{\mathbb{R}}}$.

Examples of Hamiltonian $T$ spaces satisfying the GKM hypotheses include coadjoint orbits of compact Lie groups and toric varieties. In the case of coadjoint orbits, the combinatorial description given by the GKM theorem has proved useful in the theory of equivariant Schubert calculus (for example, see [16]). These examples also have natural antisymplectic involutions, and the mod 2 results apply to these examples. The combinatorial description of the equivariant cohomology of real loci of certain toric varieties has applications to string theory (see [4]).

Thus far, we have required $M$ to be compact. However, there are many non-compact examples that nonetheless fit into this framework. For example, hypertoric varieties $[3,14,15,12,11]$ equipped with a $T \times S^{1}$ action exhibit many of the properties of compact Kähler toric varieties. For instance, like their Kähler counterparts, the $T \times S^{1}$-isotropy weights at each fixed point are pairwise linearly independent. The hypertoric varieties are also equipped with a natural antisymplectic involution, and the computation of the equivariant cohomology of the real loci has applications to the theory of hyperplane arrangements [11, Section 5]. Moreover, coadjoint orbits of affine Kac-Moody algebras equipped with an appropriate $T \times S^{1}$ action satisfy pairwise linear independence properties. Thus, the examples strongly motivate us to demonstrate GKM and mod 2 GKM theorems in the non-compact setting. 
The essential observation in this paper is that the Morse theoretic arguments given in [19] work with only slight modifications in the setting when there is a component of the moment map which is proper and bounded below. These two hypotheses allow us to apply the same local Morse theoretic arguments: the properness ensures the compactness of critical sets, and the boundedness allows us to make an inductive argument by providing a base case. This is the case for some of the examples mentioned above; for smooth hypertoric varieties, it is indeed true that there exists a component of the $T \times S^{1}$ moment map which is proper and bounded below [11]. For the coadjoint orbits of affine Kac-Moody algebras, however, the methods presented in this paper do not suffice. Different arguments must be used to give a GKM description for these examples [10]. The results in [10] are phrased in the language of cell complexes, but they also achieve a combinatorial description of equivariant cohomology. We also note that the results in this paper are stated over $\mathbb{Z}$ instead of $\mathbb{Q}$. This changes the statements of some of the technical hypotheses on the $T$-isotropy weights.

We now give a brief outline of the contents of this paper. In Section 2, we state and prove a GKM theorem in the setting of non-compact spaces in Theorem 2.11. We use this theory in Section 3 to analyze in detail the example of smooth hypertoric varieties equipped with a Hamiltonian $T^{d} \times S^{1}$ action. In particular, we give an isomorphism between the quotient description of the $T^{d} \times S^{1}$-equivariant cohomology of a hypertoric variety given in [11] with the GKM description in Theorem 3.5, and compute several examples. Further, although the $T^{d}$ action on $M$ does not satisfy the GKM hypotheses, we use a "GKM in stages" argument to give a computation of the $T^{d}$-equivariant cohomology of $M$ by using our GKM description of its $T^{d} \times S^{1}$-equivariant cohomology. In Section 4 , we state and prove a mod 2 GKM theorem in the non-compact setting, stated in Theorem 4.8. We use this to analyze the real locus of hypertoric varieties in Section 5 . In particular, we explicitly identify the isomorphism between the $T^{d} \times S^{1}$-equivariant cohomology of a hypertoric variety and the $T_{\mathbb{R}}^{d} \times \mathbb{Z}_{2}$-equivariant cohomology of its real locus in Proposition 5.1. We also mention an application of these results that is used in [11].

\section{GKM theory for non-compact spaces.}

The goal of this section is to extend results about the equivariant topology of compact symplectic Hamiltonian manifolds to situations where the manifold is not necessarily compact. We replace the compactness hypothesis by a 
hypothesis on the moment map: we require it to be proper and bounded in some component. This hypothesis ensures that we can still use components of the moment map to study the Hamiltonian manifold Morse theoretically.

Our proofs of Theorems 2.6 and 2.7 follow the outline of the arguments given in [19]. The technical heart of the argument is a lemma due to Atiyah and Bott. The hypothesis on the moment map ensures that this lemma still applies to our non-compact setting. We use this lemma, along with the Morse theory of the moment map, to show that the equivariant cohomology of $M$ injects into the equivariant cohomology of the fixed point set. We then show that the image is the same as the image of the equivariant cohomology of the (equivariant) one-skeleton, $\bar{N}$. The main result then follows as a corollary to this: we give the combinatorial description of $H_{T}^{*}(M)$, given additional hypotheses on $M^{T}$ and on $\bar{N}$.

We first present the key lemma of Atiyah and Bott. It is stated in [1, Proposition 13.4], [2, Proposition 5.3.7].

Lemma 2.1 (Atiyah-Bott). Let $\mathcal{E} \rightarrow B$ be a complex rank $\ell$ vector bundle over a compact oriented manifold $B$. Let $T$ be the compact torus $T=\left(S^{1}\right)^{d}$. Suppose that $T$ acts on $\mathcal{E}$ with fixed point set precisely $B$. Suppose further that the cohomology of $B$ has no torsion over $\mathbb{Z}$. Choose a $T$-invariant Riemannian metric on $\mathcal{E}$, and let $D$ and $S$ be the corresponding disk and sphere bundles, respectively, of $\mathcal{E}$. Then, the long exact sequence of the pair $(D, S)$ splits into short exact sequences

$$
0 \longrightarrow H_{T}^{*}(D, S ; \mathbb{Z}) \longrightarrow H_{T}^{*}(D ; \mathbb{Z}) \longrightarrow H_{T}^{*}(S ; \mathbb{Z}) \longrightarrow 0 .
$$

Remark 2.2. An alternative statement of this lemma is that the $T$ equivariant Euler class of the bundle $\mathcal{E}$ is not a zero divisor.

Remark 2.3. The hypothesis that the cohomology of $B$ has no torsion over $\mathbb{Z}$ can be relaxed to the hypothesis that it has no 2 -torsion when we take the coefficient ring to be $\mathbb{Z}_{2}$, and can be removed entirely if we take coefficient $\operatorname{ring} \mathbb{Q}$.

We now turn our attention to finite-dimensional Hamiltonian $T$ spaces. Suppose that a torus $T$ acts on a symplectic manifold $M$ in a Hamiltonian fashion. Then, components $\mu^{\xi}$ of the moment map $\mu: M \rightarrow \mathfrak{t}^{*}$ are MorseBott functions on $M$. We call a component generic if the critical set is precisely the fixed point set. This is true of an open dense set of directions $\xi \in \mathfrak{t}$. When we assume that a generic component is proper, then the connected components of the fixed point set are compact. Thus, we may 
use Lemma 2.1 to study the normal bundles to these fixed point sets to prove the following proposition.

Proposition 2.4. Let a torus $T$ act on a symplectic manifold $M$ with moment map $\mu: M \rightarrow \mathfrak{t}^{*}$. Suppose that some generic component $f:=\mu^{\xi}$ is proper and bounded below. Let $c$ be a critical value of $f$. Let $\Sigma_{c}$ be the component of $\Sigma:=M^{T}$ with $\mu^{\xi}\left(\Sigma_{c}\right)=c$, and assume that the cohomology of $\Sigma_{c}$ has no torsion over $\mathbb{Z}$. For small enough $\varepsilon>0$, let $M_{c}^{ \pm}:=f^{-1}(-\infty, c \pm \varepsilon)$. Then, the long exact sequence of the pair $\left(M_{c}^{+}, M_{c}^{-}\right)$splits into short exact sequences

$$
0 \longrightarrow H_{T}^{*}\left(M_{c}^{+}, M_{c}^{-} ; \mathbb{Z}\right) \longrightarrow H_{T}^{*}\left(M_{c}^{+} ; \mathbb{Z}\right) \stackrel{k^{*}}{\longrightarrow} H_{T}^{*}\left(M_{c}^{-} ; \mathbb{Z}\right) \longrightarrow 0 .
$$

Moreover, the restriction from $H_{T}^{*}\left(M_{c}^{+} ; \mathbb{Z}\right)$ to $H_{T}^{*}\left(\Sigma_{c} ; \mathbb{Z}\right)$ induces an isomorphism from the kernel of $k^{*}$ to the classes of $H_{T}^{*}\left(\Sigma_{c} ; \mathbb{Z}\right)$ that are multiples of $\tau_{c}$ the equivariant Euler class of the negative normal bundle to $\Sigma_{c}$.

Remark 2.5. Note that in the proof, we implicitly assume that the component $\Sigma_{c}$ of $\Sigma$ that maps to the critical value $c$ is connected. If this is not the case, an analogous statement holds, using a virtual equivariant Euler class. The reason that care is needed in that case is that the negative normal bundle need not have the same dimension on the different connected components of $\Sigma_{c}$.

Proof. This argument appears in [19]. Let $D_{c}$ and $S_{c}$ denote the disc and sphere bundles of the negative normal bundle to the fixed set $\Sigma_{c}$. Using the retraction of the pair $\left(M_{c}^{+}, M_{c}^{-}\right)$to the pair $\left(D_{c}, S_{c}\right)$ and the Thom isomorphism, we get the commutative diagram

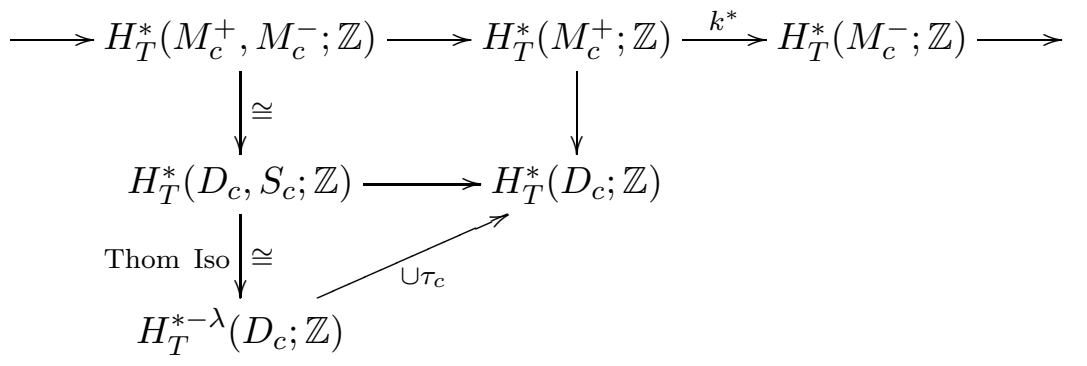

By the Atiyah-Bott Lemma, the cup product with $\tau_{c}$ is injective; therefore, the top long exact sequence splits into short exact sequences. The proposition follows by a diagram chase. 
Using this proposition, we prove by an inductive argument that the equivariant cohomology of $M$ injects into the equivariant cohomology of the fixed point set $\Sigma$. In order to start the induction, we now add the hypothesis that a generic component of the moment map is not only proper, but also bounded below.

Theorem 2.6. Let a torus $T$ act on a symplectic manifold $M$ with moment map $\mu: M \rightarrow \mathfrak{t}^{*}$. Suppose that some generic component $f:=\mu^{\xi}$ is proper and bounded below. Suppose that $\Sigma=M^{T}$ has only finitely many connected components. Let $\imath: \Sigma \rightarrow M$ be the inclusion of the fixed point set into $M$. Then, the pullback map

$$
\imath^{*}: H_{T}^{*}(M ; \mathbb{Z}) \rightarrow H_{T}^{*}(\Sigma ; \mathbb{Z})
$$

is injective.

Proof. Choose $\xi$ with $f=\mu^{\xi}$ generic, proper, and bounded below. The critical sets of $f$ are precisely the connected components of $\Sigma$. Thus, by assumption on $\Sigma$, there are only finitely many critical values of $f$. Order these critical values as $c_{1}<c_{2}<\cdots<c_{m}$, and let $\Sigma_{c_{1}}, \ldots, \Sigma_{c_{m}}$ denote the corresponding critical submanifolds. These critical submanifolds are compact, since $f$ is proper. Let $\Sigma_{c_{i}}^{ \pm}:=M_{c_{i}}^{ \pm} \cap \Sigma$. We now proceed by induction on the critical values.

Let $\varepsilon>0$ be smaller than any of the values $c_{i}-c_{i-1}$. The injectivity result is true for $M_{c_{1}}^{+}$, as it is equivariantly homotopic to $\Sigma_{c_{1}}$. Now assume by induction that it is true for $M_{c_{i}}^{-}$. We will prove that it is true for $M_{c_{i}}^{+}$. Note that $M_{c_{i}}^{-}$is homotopy equivalent to $M_{c_{i-1}}^{+}$. We have the long exact sequence of the pair $\left(M_{c_{i}}^{+}, M_{c_{i}}^{-}\right)$, but by Proposition 2.4, this splits into short exact sequences. Thus, we have a commutative diagram

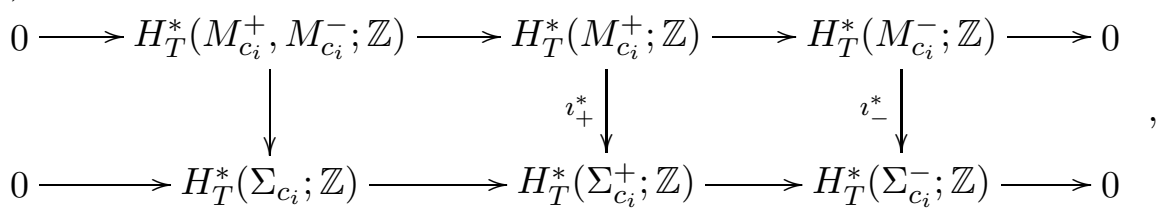

where we identify $H_{T}^{*}\left(\Sigma_{c_{i}}^{+}, \Sigma_{c_{i}}^{-} ; \mathbb{Z}\right) \cong H_{T}^{*}\left(\Sigma_{c_{i}} ; \mathbb{Z}\right)$. The left vertical arrow is an injection, induced by the Thom isomorphism, and the right vertical arrow is an injection by the induction hypothesis. A simple diagram chase shows that the middle vertical arrow must also be an injection. Since there are only finitely many critical values, the result now follows by induction. 
Since $T$ is acting trivially on $\Sigma$, the $\operatorname{ring} H_{T}^{*}(\Sigma ; \mathbb{Z})$ is isomorphic to the ring $H^{*}(\Sigma ; \mathbb{Z}) \otimes H_{T}^{*}(p t ; \mathbb{Z})$. In general, $H_{T}^{*}(\Sigma ; \mathbb{Z})$ is easier to compute than $H_{T}^{*}(M ; \mathbb{Z})$. Thus, in order to compute $H_{T}^{*}(M ; \mathbb{Z})$ as a ring, it now suffices to describe the image in $H_{T}^{*}(\Sigma ; \mathbb{Z})$. We will now show that in fact, the image of $H_{T}^{*}(M ; \mathbb{Z})$ is the same as the image of the equivariant cohomology of a certain subset of $M$.

Let $N$ denote the subset of $M$ given by

$$
N:=\{x \in M \mid \operatorname{codim}(\operatorname{Stab}(x))=1\} .
$$

Thus, $N$ consists of the points in $M$ whose $T$ orbit is exactly onedimensional. We now define the one-skeleton of $M$ to be the closure of $N$. That is, it is the set

$$
\bar{N}:=\{x \in M \mid \operatorname{codim}(\operatorname{Stab}(x)) \leq 1\} .
$$

We have the diagram of inclusions

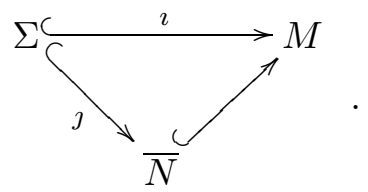

The next theorem states that the image of $H_{T}^{*}(M ; \mathbb{Z})$ is the same as the image of $H_{T}^{*}(\bar{N} ; \mathbb{Z})$ in $H_{T}^{*}(\Sigma ; \mathbb{Z})$. It is a non-compact version of a theorem of Tolman and Weitsman [19, Theorem 1]. As above, the compactness hypothesis is replaced by the hypothesis that some generic component of the moment map be proper and bounded. Note that our theorem holds with $\mathbb{Z}$ coefficients in contrast to $[19$, Theorem 1$]$, which is stated for $\mathbb{Q}$ coefficients. To achieve this, we have added an assumption on the $T$ weights on the negative normal bundle.

Theorem 2.7. Let a torus $T$ act on a symplectic manifold $M$ with moment map $\mu: M \rightarrow \mathfrak{t}^{*}$. Suppose that some generic component $f:=\mu^{\xi}$ is proper and bounded below. Suppose that $\Sigma:=M^{T}$ has only finitely many connected components. Suppose further that the distinct weights of the $T$ action on the negative normal bundle, with respect to $f$, to any fixed point component are pairwise relatively prime in $H_{T}^{*}(p t ; \mathbb{Z})$. Then, in the diagram in equivariant cohomology, induced by the inclusions (2.2),

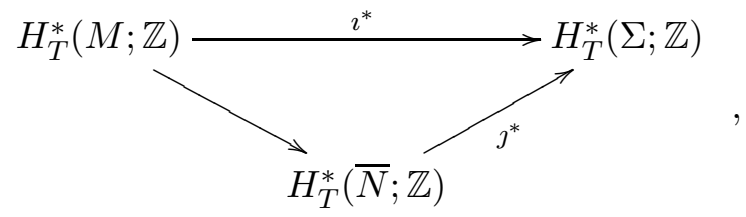


the image of $\imath^{*}$ is equal to the image of $\jmath^{*}$ in $H_{T}^{*}(\Sigma ; \mathbb{Z})$.

Remark 2.8. Note that if $T=S^{1}$, we have $\bar{N}=M$, and the theorem automatically holds.

Proof. We proceed by induction on the critical values $c_{1}<c_{2}<\cdots<c_{m}$ of $f:=\mu^{\xi}$. We first set up our notation. Let $c$ be one of the critical values of $f$. Define the sets $\bar{N}_{c}^{ \pm}:=\bar{N} \cap M_{c}^{ \pm}$. Then, we have inclusions

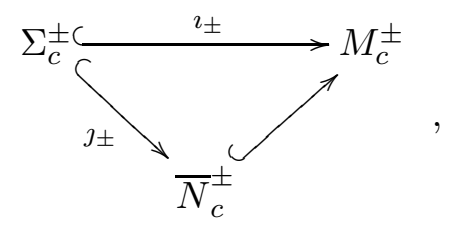

which induce, in equivariant cohomology,

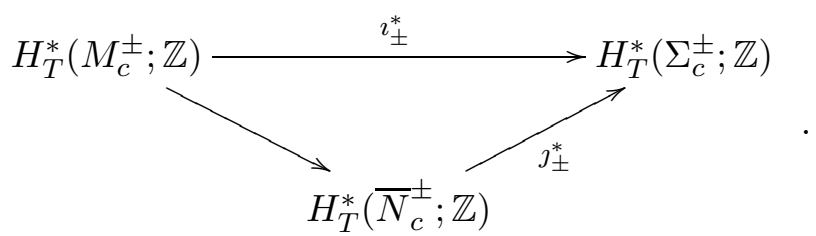

The base case consists of analyzing these diagrams for the minimum critical value $c_{1}$. In this case, $M_{c_{1}}^{-}$and $N_{c_{1}}^{-}$are empty, and $M_{c_{1}}^{+}$and $N_{c_{1}}^{+}$both equivariantly retract onto $\Sigma_{c_{1}}$. Thus, both $\imath_{+}^{*}$ and $\jmath_{+}^{*}$ are isomorphisms, and therefore have the same image.

Assume now by induction that the statement holds for $M_{c_{i-1}}^{+}$. Let $r$ denote the natural restriction from $i m\left(\jmath_{+}^{*}\right) \subseteq H_{T}^{*}\left(\Sigma_{c_{i}}^{+} ; \mathbb{Z}\right)$ to $H_{T}^{*}\left(\Sigma_{c_{i}}^{-} ; \mathbb{Z}\right)$. Note that the image of $r$ is contained in $i m\left(J_{-}^{*}\right)$. By abuse of notation, we will let $\operatorname{ker}(r)$ denote the inverse image inside $H_{T}^{*}\left(\Sigma_{c_{i}} ; \mathbb{Z}\right) \cong H_{T}^{*}\left(\Sigma_{c_{i}}^{+}, \Sigma_{c_{i}}^{-} ; \mathbb{Z}\right)$ of the kernel of $r$, using the short exact sequence of the pair $\left(\Sigma_{c_{i}}^{+}, \Sigma_{c_{i}}^{-}\right)$. Thus, we have a commutative diagram

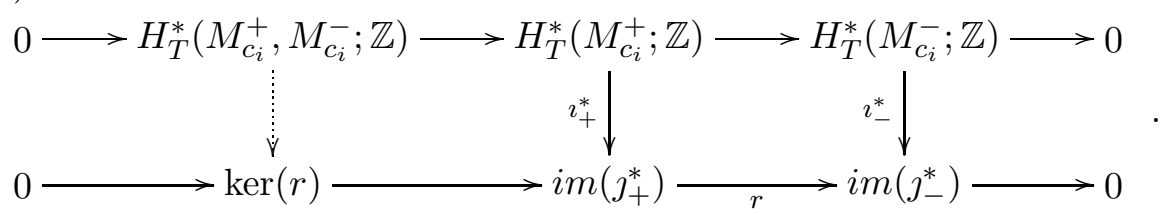

The map $\imath_{-}^{*}$ is a surjection, by the inductive hypothesis. To show that $\imath_{+}^{*}$ is a surjection, it suffices to show that the dotted vertical arrow is a surjection. That $\imath_{+}^{*}$ is a surjection then follows by a diagram chase. 
We first recall a fact about Euler classes. Suppose $T$ acts on a complex vector bundle $\mathcal{E}$ over a manifold $\Sigma$, with fixed point set precisely $\Sigma$. Decompose $\mathcal{E}$ into the direct sum of bundles $\mathcal{E}_{\alpha}$, where $T$ acts on $\mathcal{E}_{\alpha}$ by weight $\alpha \in \mathfrak{t}_{\mathbb{Z}}^{*}$. Assume that weights $\alpha$ are distinct and pairwise relatively prime in $H_{T}^{2}(p t ; \mathbb{Z}) \cong \mathfrak{t}_{\mathbb{Z}}^{*}$. Let $\tau_{\alpha}$ be the equivariant Euler class of the subbundle $\mathcal{E}_{\alpha}$. Then, if $y \in H_{T}^{*}(\Sigma ; \mathbb{Z})$ is a multiple of $\tau_{\alpha}$ for each $\alpha$, then $y$ is a multiple of the product of the $\tau_{\alpha}$. This follows from the proof of [19, Lemma 3.2]. Although their Lemma is stated for $\mathbb{Q}$ coefficients, the argument goes through given our assumption of relative primality of the weights.

We now characterize $\operatorname{ker}(r)$. Suppose $\eta$ is a class in $H_{T}^{*}\left(\bar{N}_{c_{i}}^{+} ; \mathbb{Z}\right)$ such that its restriction to $H_{T}^{*}\left(\Sigma_{c_{i}}^{-} ; \mathbb{Z}\right)$ is zero; that is, $r \circ j_{+}^{*}(\eta)=0$. Let $\nu=\oplus_{\alpha} \nu_{\alpha}$ be the $T$-invariant decomposition of the negative normal bundle to $\Sigma_{c_{i}}$, where the weights $\{\alpha\}$ are distinct. Let $N_{\alpha}$ be the component of the oneskeleton $N$ corresponding to the weight $\alpha$ and such that the closure contains $\Sigma_{c_{i}}$. The closure $\bar{N}_{\alpha}$ is a symplectic manifold, and the restriction of $\eta$ to $\bar{N}_{\alpha}^{+}:=\bar{N}_{\alpha} \cap M_{c_{i}}^{+}$has the property that it vanishes when restricted to $\bar{N}_{\alpha}^{-}:=\bar{N}_{\alpha} \cap M_{c_{i}}^{-}$. This is because $\eta$ vanishes when restricted to $\Sigma_{c_{i}}^{-}$(by injectivity for $\left.\bar{N}_{\alpha}\right)$. By Proposition 2.4 applied to the pair $\left(\bar{N}_{\alpha}^{+}, \bar{N}_{\alpha}^{-}\right)$, we may conclude that $\eta$ restricted to $\Sigma_{c_{i}}$ must be a multiple of each $\tau_{\alpha}$. By assumption, any two distinct $T$ weights occurring in the negative normal bundle to $\Sigma_{c_{i}}$ are relatively prime in $H_{T}^{*}(p t ; \mathbb{Z})$. Hence, by the fact recalled in the previous paragraph, the restriction of $\eta$ to $H_{T}^{*}\left(\Sigma_{c_{i}} ; \mathbb{Z}\right)$ has to be a multiple of the product of the $\tau_{\alpha}$, which is the equivariant Euler class of the negative normal bundle to $\Sigma_{c_{i}}$.

We now show that the left vertical arrow in the diagram (2.3) is a surjection. We have shown that any element in $\operatorname{ker}(r)$ is, when restricted to $\Sigma_{c_{i}}$, a multiple of the equivariant Euler class $\tau_{c_{i}}$ of the negative normal bundle to $\Sigma_{c_{i}}$. On the other hand, any class in $H_{T}^{*}\left(\Sigma_{c}\right)$ which is a multiple of $\tau_{c}$ is the image of an element in $H_{T}^{*}\left(M^{+}, M^{-}\right)$by Proposition 2.4. Hence, the left vertical arrow is surjective, and the surjectivity of $\imath_{-}^{*}$ follows by the five lemma.

Theorem 2.7 tells us that it suffices to identify the image of $\jmath^{*}$ to find a description of $H_{T}^{*}(M)$. We will now place stronger hypotheses on the fixed point set $\Sigma$ and the one-skeleton $\bar{N}$ so that the image of $\jmath^{*}$ has a simple combinatorial description. We make the following definition.

Definition 2.9. Let $M$ be a symplectic manifold equipped with a Hamiltonian $T$ action. We say that the action is GKM if $M^{T}$ consists of finitely 
many isolated points, and the $T$-isotropy weights $\alpha_{i, p}$ at a given fixed point are pairwise relatively prime in $H_{T}^{*}(M ; \mathbb{Z})$.

Henceforth, we assume that our action is GKM. Thus, each component of $\Sigma$ is an isolated point, and all equivariant Euler classes are elements of $H_{T}^{*}(p t ; \mathbb{Z})$, given as products of the relevant isotropy weights. Moreover, if the moment map is proper and bounded below in some component, the one-skeleton is a union of copies of $\mathbb{C} P^{1}$ and $\mathbb{C}$, intersecting in fixed points. When $M$ is compact, the pairwise relative primality of the isotropy weights is equivalent to the one-skeleton being two-dimensional [9]. The same holds for GKM actions in the presence of a moment map that is proper and bounded below in some component, by a symplectic cutting argument.

We now associate a graph $\Gamma$ to the GKM action on $M$ that encodes the information necessary to compute the equivariant cohomology of $M$. We call this the GKM graph. The vertices $V$ of $\Gamma$ are the fixed points $M^{T}$. The edges $E$ of $\Gamma$ correspond to the embedded $\mathbb{C} P^{1}$ 's. That is, we include an edge between two fixed points precisely when they are the two fixed points of a $\mathbb{C} P^{1}$ in the one-skeleton. Each edge $e \in E$ is labeled with the weight $\alpha_{e}$ of the torus action on that copy of $\mathbb{C} P^{1}$. Notice that the $\mathbb{C}$ 's in the one-skeleton equivariantly retract, and therefore do not contribute to the cohomology of the one-skeleton. Thus, we do not record this information in the graph $\Gamma$.

The computation of the cohomology of the one-skeleton for a GKM action now boils down to the computation of the $T$-equivariant cohomology of $\mathbb{C} P^{1}$. For the proof of the following Lemma, see, for instance, [10].

Lemma 2.10. Suppose $T$ acts linearly and non-trivially on $\mathbb{C} P^{1}$ with weight $\alpha$. Then, the inclusion of the fixed points $\left(\mathbb{C} P^{1}\right)^{T}=\{N, S\}$ into $\mathbb{C} P^{1}$ induces an injection $\imath^{*}: H_{T}^{*}\left(\mathbb{C} P^{1} ; \mathbb{Z}\right) \rightarrow H_{T}^{*}(\{N, S\} ; \mathbb{Z})$, with image

$$
\begin{gathered}
\imath^{*}\left(H_{T}^{*}\left(\mathbb{C} P^{1}\right) ; \mathbb{Z}\right)=\left\{(f, g) \in H_{T}^{*}(\{N\} ; \mathbb{Z}) \oplus H_{T}^{*}(\{S\} ; \mathbb{Z}) \mid(f-g)\right. \\
\cong 0(\bmod \alpha)\} .
\end{gathered}
$$

Motivated by this lemma, we now define the graph cohomology of $\Gamma$ to be

$$
\begin{aligned}
& H^{*}(\Gamma, \alpha):=\left\{\begin{array}{l|c}
f: V \rightarrow H_{T}^{*}(p t ; \mathbb{Z}) & \begin{array}{c}
f(p)-f(q) \equiv 0\left(\bmod \alpha_{e}\right) \\
\text { for every edge } e=(p, q)
\end{array}
\end{array}\right\} \\
& \subseteq H_{T}^{*}(V ; \mathbb{Z})
\end{aligned}
$$


Since the one-skeleton consists of $\mathbb{C} P^{1}$ 's (and equivariantly retractable $\mathbb{C}$ 's) intersecting at fixed points, a Mayer-Vietoris type argument shows that the image of the cohomology of the one-skeleton under $\jmath^{*}$ is precisely the graph cohomology. This, combined with Theorem 2.7, yields the following theorem.

Theorem 2.11. Let a torus $T$ act on a symplectic manifold $M$ with moment map $\mu: M \rightarrow \mathfrak{t}^{*}$. Suppose that some generic component $f:=\mu^{\xi}$ is proper and bounded below. Suppose that $\Sigma=M^{T}$ consists of only finitely many isolated points, and that the $T$-isotropy weights at $p \in \Sigma$ are pairwise relatively prime in $H_{T}^{*}(p t ; \mathbb{Z})$. Then, under the map $\imath^{*}$, the equivariant cohomology $H_{T}^{*}(M ; \mathbb{Z})$ maps isomorphically onto $H^{*}(\Gamma, \alpha)$.

\section{Examples: hypertoric varieties.}

In this section, we present the examples that motivated the work in this paper. These are the hypertoric varieties studied in $[3,11,14,15]$. Like their Kähler counterparts, hypertoric varieties come equipped with natural $T^{d}$ actions. However, it is important to note that the GKM hypotheses only hold for the $T^{d} \times S^{1}$ action on hypertoric varieties, where the $S^{1}$ action is an extra piece of structure on hypertoric varieties not present in the Kähler versions. This will be explained in detail below. Throughout this section, we take the coefficient ring $R=\mathbb{Z}$.

We first set some notation in order to facilitate discussion of the examples. For details, we refer the reader to $[3,11]$. Let $T^{n}$ be the real $n$-dimensional torus acting on $\mathbb{C}^{n}$, with induced action on $\mathbb{H}^{n} \cong T^{*} \mathbb{C}^{n}$ given by $t(z, w)=\left(t z, t^{-1} w\right)$. Let $\left\{a_{i}\right\}_{1 \leq i \leq n}$ be non-zero primitive integer vectors in $\mathfrak{t}^{d} \cong \mathbb{R}^{d}$ and let $\left\{\varepsilon_{i}\right\}$ be the standard basis for $\mathfrak{t}^{n} \cong \mathbb{R}^{n}$, dual to $\left\{h_{i}\right\}$ the standard basis for $\left(\mathfrak{t}^{n}\right)^{*}$. Define the map $\beta: \mathfrak{t}^{n} \longrightarrow \mathfrak{t}^{d}$ by setting $\beta\left(\varepsilon_{i}\right)=a_{i}$, This map fits into an exact sequence

$$
0 \longrightarrow \mathfrak{t}^{k} \stackrel{\iota}{\longrightarrow} \mathfrak{t}^{n} \stackrel{\beta}{\longrightarrow} \mathfrak{t}^{d} \longrightarrow 0
$$

where $\mathfrak{t}^{k}:=\operatorname{ker}(\beta)$. Exponentiating yields a subtorus $T^{k}$ of $T^{n}$.

The action of $T^{n}$ on $\mathbb{H}^{n}$ is hyperhamiltonian, and so the $T^{k}$ action is also hyperhamiltonian. We denote by $M$ the hyperkähler reduction of $\mathbb{H}^{n}$ by the subtorus $T^{k}$ at $(\lambda, 0) \in\left(\mathfrak{t}^{k}\right)^{*} \oplus\left(\mathfrak{t}_{\mathbb{C}}^{k}\right)^{*}$, which we assume is a regular value. This is the hyperkähler analogue of the Kähler toric variety $X=\mathbb{C}^{n} /{ }_{\lambda} T^{k}$. The reduction $M$ has a residual action of $T^{d}$ with hyperkähler moment map, denoted $\mu=\mu_{\mathbb{R}} \oplus \mu_{\mathbb{C}}$. Note that the choice of subtorus $T^{k} \subseteq T^{n}$ and a lift 
$\tilde{\lambda}$ of $\lambda$ amounts to choosing an arrangement $\mathcal{H}$ of cooriented, affine, rational hyperplanes $\left\{H_{i}\right\}_{i=1}^{n}$, where the $i$-th hyperplane is

$$
H_{i}=\left\{x \in\left(\mathfrak{t}^{d}\right)^{*} \mid\left\langle x, a_{i}\right\rangle=\left\langle-\tilde{\lambda}, \varepsilon_{i}\right\rangle\right\} .
$$

The coorientation comes from knowing for which $x$, we have $\left\langle x, a_{i}\right\rangle>0$. To record the coorientations, we define the half-spaces

$$
\begin{gathered}
F_{i}=\left\{x \in\left(\mathfrak{t}^{d}\right)^{*} \mid\left\langle x, a_{i}\right\rangle \geq\left\langle-\tilde{\lambda}, \varepsilon_{i}\right\rangle\right\} \\
\quad \text { and } \\
G_{i}=\left\{x \in\left(\mathfrak{t}^{d}\right)^{*} \mid\left\langle x, a_{i}\right\rangle \leq\left\langle-\tilde{\lambda}, \varepsilon_{i}\right\rangle\right\},
\end{gathered}
$$

which intersect in the hyperplane $H_{i}$. In our examples, we assume that the half-spaces $F_{i}$ intersect in a non-empty bounded polytope $\Delta=\cap_{i=1}^{n} F_{i}$. See Figure 1 for an example. This polytope $\Delta$ is exactly the image under $\mu_{\mathbb{R}}$ of the Kähler toric variety $X=\mathbb{C}^{n} / /{ }_{\lambda} T^{k}$.

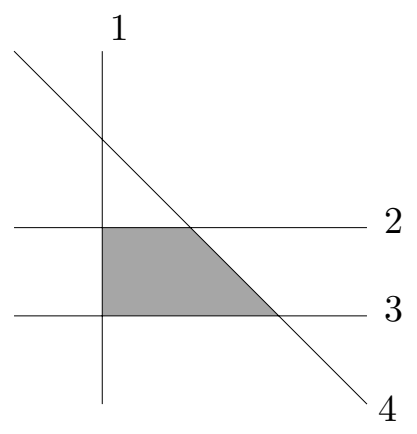

Figure 1: A simple example of a hypertoric variety of real dimension 8 obtained by reducing $\mathbb{H}^{4}$ by $T^{2}$. We label the hyperplane $H_{i}$ by the index $i$. The region $\Delta$ is shaded. The corresponding Kähler toric variety is a Hirzebruch surface.

In the case of hypertoric varieties, there is an additional residual Hamiltonian $S^{1}$ action descending from the action of $S^{1}$ on the cotangent bundle $T^{*} \mathbb{C}^{n}$ that rotates the fibers with weight 1 . Since this restricts to the trivial action on the zero section $\mathbb{C}^{n}$, the $S^{1}$ action is trivial on the Kähler toric variety. Hence, this action is a new feature of hypertoric varieties, and it turns out that this new $S^{1}$ action is essential for the GKM description of the $T^{d} \times S^{1}$ equivariant cohomology of $M$. We denote the moment map for the extra $S^{1}$ action by $\psi$. 
We begin by showing that the hypertoric varieties built above by the hyperKähler Delzant construction satisfy the hypotheses of Theorem 2.11. We always assume that the affine, rational, cooriented hyperplane arrangement $\mathcal{H}$ is smooth in the sense of [11], which implies that the hypertoric variety $M$ associated to $\mathcal{H}$ is smooth. (In particular, this means that the arrangement is simple: every subset of $m$ hyperplanes intersect in codimension $m$.) Moreover, we also assume that the polytope $\Delta$ is non-empty and bounded in $\left(\mathfrak{t}^{d}\right)^{*}$. We first show that there is a component of the moment map which is proper, bounded, and Morse.

Lemma 3.1. Let $M$ be the hypertoric variety associated to an affine, cooriented, rational, smooth hyperplane arrangement $\mathcal{H}$ such that $\Delta=\cap_{i} F_{i}$ is non-empty and bounded. Let $(\mu, \psi)$ be the $T^{d} \times S^{1}$ moment map on $M$. Then there is a component of $(\mu, \psi)$ which is proper, bounded, and Morse.

Proof. Since we assume that $\Delta$ is bounded, by [11, Proposition 1.3], the residual $S^{1}$ moment map $\psi$ is proper. Moreover, since the original $S^{1}$ moment map $\tilde{\psi}$ on $T^{*} \mathbb{C}^{n}$ is given by a norm-square of the cotangent vector, it is bounded below by 0 . Hence, the moment map $\psi$ on the quotient is also bounded below. Now, consider the $T^{d} \times S^{1}$ moment map $(\mu, \psi)$, with values in $\left(\mathfrak{t}^{d}\right)^{*} \times \mathbb{R} \cong \mathbb{R}^{d+1}$. We have just shown that the component $(\mu, \psi)^{\xi}=\psi$ of this moment map is proper and bounded below. By taking a small enough perturbation of $\xi$, we obtain a generic component of the moment map which is proper, bounded, and also Morse.

We must now show that the $T^{d} \times S^{1}$ fixed points on $M$ is a finite collection of isolated points, and that the isotropy weights are relatively prime. We set the following notation. The hyperplanes $\left\{H_{i}\right\}$ divide $\left(\mathfrak{t}^{d}\right)^{*} \cong \mathbb{R}^{d}$ into a finite family of closed, convex polyhedra

$$
\Delta_{A}=\left(\cap_{i \notin A} F_{i}\right) \cap\left(\cap_{i \in A} G_{i}\right),
$$

indexed by subsets $A \subseteq\{1, \ldots, n\}$. For each $A \subseteq\{1, \ldots, n\}$, let

$$
M_{A}=\mu_{\mathbb{R}}^{-1}\left(\Delta_{A}\right) \cap \mu_{\mathbb{C}}^{-1}(0) .
$$

This is a Kähler submanifold of $M$ with respect to $\omega_{\mathbb{R}}$, and is the (possibly non-compact) Kähler toric variety associated to $\Delta_{A}[3,6.5]$.

Proposition 3.2. Let $M$ satisfy the hypotheses of Lemma 3.1. Then, the action of $T^{d} \times S^{1}$ on $M$ is GKM. 
Proof. We will need the following facts, all of which may be found in [11]. Since the $\mathbb{C}$ moment map $\mu_{\mathbb{C}}$ is $S^{1}$-equivariant (where $S^{1}$ acts on $\mathfrak{t}_{\mathbb{C}}^{*}$ by the standard rotation action), the $S^{1}$-fixed points of $M$ must lie in $\mu_{\mathbb{C}}^{-1}(0)=$ $\bigcup_{A} M_{A}$. On each $M_{A}$, the torus $T^{d}$ acts in a Hamiltonian fashion with respect to $\omega_{\mathbb{R}}$ with moment map $\left.\mu_{\mathbb{R}}\right|_{M_{A}}$ and image $\Delta_{A}$. Moreover, on each $M_{A}$, the extra $S^{1}$ action acts as a subtorus of $T^{d}$, determined combinatorially by $A$.

Since we are looking for $T^{d} \times S^{1}$-fixed points, the fact that all the $S^{1}$-fixed points are contained in $\mu_{\mathbb{C}}^{-1}(0)$ allows us to restrict our attention to the toric varieties $M_{A}$. Since the $M_{A}$ are usual toric varieties, we find immediately that $M^{T^{d} \times S^{1}}$ is a subset of the points in $\mu_{\mathbb{C}}^{-1}(0)$ corresponding to the vertices $v \in\left(\mathfrak{t}^{d}\right)^{*}$ of the polyhedral complex defined by $\mathcal{H}$. On the other hand, we know from the description of $M^{S^{1}}$ in [11] that each such point in $\mu_{\mathbb{C}}^{-1}(0)$ corresponding to a vertex $v$ is also fixed by $S^{1}$. Hence, the fixed points $M^{T^{d} \times S^{1}}$ are isolated, with images under $\mu_{\mathbb{R}}$ exactly the vertices $v=\cap_{i \in I} H_{i}$ in the hyperplane arrangement. In particular, $\left|M^{T^{d} \times S^{1}}\right|$ is finite.

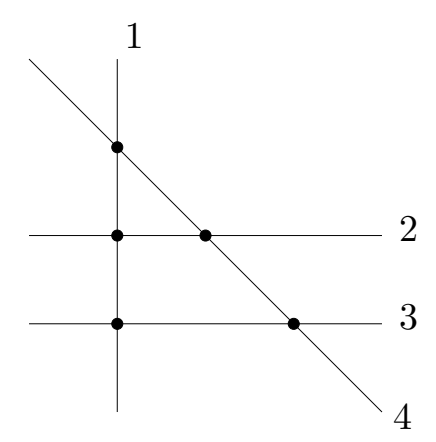

Figure 2: The $T^{d} \times S^{1}$-fixed points are mapped to the vertices of the hyperplane arrangement.

We must now check that the $T^{d} \times S^{1}$ weights at a given fixed point $p$ are pairwise relatively prime in $H_{T^{d} \times S^{1}}^{*}(p t)$. Let $I \subset\{1,2, \ldots, n\}$ be a subset of size $d$ such that $\cap_{i \in I} H_{i} \neq \emptyset$. Since $\mathcal{H}$ is simple, the intersection is a single vertex $v$. Let $p$ be the $T^{d} \times S^{1}$-fixed point mapping to the vertex $v=\mu_{\mathbb{R}}(p)$. We wish to decompose $T_{p} M$ under the $T^{d} \times S^{1}$-isotropy action into a sum of 1-dimensional pieces. Since the arrangement $\mathcal{H}$ is simple, there are exactly $2 d$ edges coming out of the vertex $v$, with two edges for each $i \in I$. See Figure 3. Each edge $e$ defines part of a polytope $\Delta_{A}$ corresponding to a subvariety $M_{A}$ containing $p$. Since $M_{A}$ is a standard toric variety, there 
exists a 1-dimensional weight space in $T_{p} M_{A} \subseteq T_{p} M$ with $T^{d}$ weight $\alpha_{e}$, where $\alpha_{e}$ is the weight corresponding to that edge in $\left(\mathfrak{t}^{d}\right)^{*}$. Since all the weights $\alpha_{e}$ are distinct in $\left(\mathfrak{t}^{d}\right)^{*}$, we get a $T^{d}$ decomposition

$$
T_{p} M \cong \oplus_{i=1}^{2 d} \mathbb{C}_{\alpha_{e_{i}}} .
$$

This is also a $T^{d} \times S^{1}$ decomposition because the $S^{1}$ commutes with $T^{d}$.

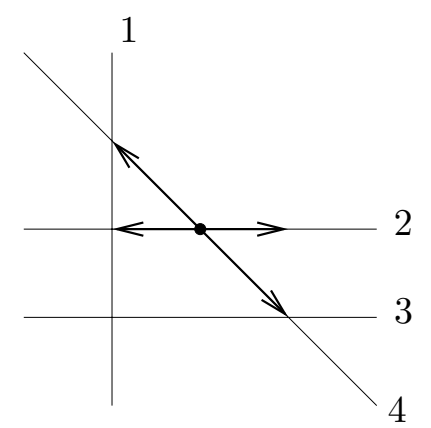

Figure 3: Each edge coming out of a vertex corresponds to a 1-dimensional subspace in $T_{p} M$. Here, the dimension of the hypertoric variety $M$ is 4 , and there are 4 distinct $T^{d}$ weights.

We must now show that the $T^{d} \times S^{1}$ weights are pairwise linearly independent. The hyperplane arrangement $\mathcal{H}$ is simple, so for $v=\cap_{i \in I} H_{i}$ as above, the collection $\left\{a_{i}\right\}_{i \in I}$ form a $\mathbb{Z}$ basis of $\left(\mathfrak{t}^{d}\right)_{\mathbb{Z}}$. Recall that for each hyperplane $H_{i}$, we have two weights $\alpha_{e}$ corresponding to $H_{i}$ in the decomposition (3.3). These are the two edges that do not lie in the hyperplane $H_{i}$. Let $\left\{\alpha_{e_{i}}\right\}$ be a collection of $T^{d}$ weights in $T_{p} M$ with $\left|\left\{\alpha_{e_{i}}\right\}\right|=d$, where we have chosen a single weight corresponding to each $H_{i}$. Then, the fact that $\mathcal{H}$ is simple implies that the collection of $T^{d}$ weights $\left\{\alpha_{e_{i}}\right\}$ is linearly independent over $\mathbb{Z}$, so, in particular, pairwise relatively prime over $H_{T^{d}}^{*}(p t ; \mathbb{Z})$. We may immediately conclude that for $T^{d} \times S^{1}$ edge weights $\alpha_{e_{i}}, \alpha_{e_{j}}$ (here, we abuse notation and use $\alpha_{e}$ to denote both $T^{d}$ and $T^{d} \times S^{1}$ weights) are pairwise relatively prime over $H_{T^{d} \times S^{1}}^{*}(p t ; \mathbb{Z})$ if $e_{i}, e_{j}$ lie on different hyperplanes.

It remains to deal with the case when two weights $\alpha_{e}, \alpha_{e l}$ correspond to the same hyperplane. In this case, as $T^{d}$ weights, they are negative multiples of one another. Hence, to get relative primality, we must compare their $S^{1}$ weights. In order to compute this $S^{1}$ weight on a given $\mathbb{C}_{\alpha_{e}}$, we use the fact that the action of $S^{1}$ on each $M_{A}$ is that of a subtorus (depending 
combinatorially on $A$ ) of $T^{d}$. It follows from the computation in [11] that the $S^{1}$ weight on $\mathbb{C}_{\alpha_{e}}$ is given by $\left\langle\alpha_{e},-\sum_{i \in A} a_{i}\right\rangle \in \mathbb{Z}$ for $\Delta_{A}$ containing both the vertex $v$ and the edge $e$. Although the choice of $A$ here is not unique, the weight is well-defined. For if $\alpha_{e}$ is an edge weight for $M_{A}$ and $M_{A^{\prime}}$, where $A$ and $A^{\prime}$ differ by a single $i$, then $a_{i}$ is necessarily in the annihilator of $\alpha_{e}$. See Figure 4. By a simple inductive argument, we conclude that the pairing above remains constant for different choices of $M_{A}$.

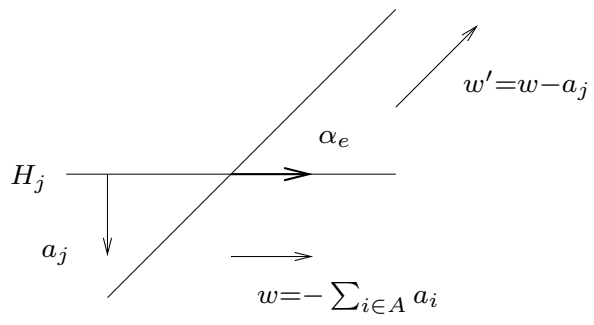

Figure 4: The computation of the $S^{1}$ weight for the edge $e$. The pairing $\left\langle\alpha_{e},-\sum_{i \in A} a_{i}\right\rangle$ is well-defined since for two adjacent regions, the corresponding vectors $v$ and $v^{\prime}$ differ by a vector $a_{j}$ perpendicular to $\alpha_{e}$.

To see that $\alpha_{e}, \alpha_{e^{\prime}}$ are relatively prime, it suffices to check that the $S^{1}$ weights are not negative multiples of each other. Let $A$ be such that $\Delta_{A}$ contains $v$ and $e$. Let $a_{j}$ define the (unique) hyperplane $H_{j}, j \in I$, for which $\alpha_{e}, \alpha_{e^{\prime}}$ do not lie on $H_{j}$. Without loss of generality, we assume $\left\langle\alpha_{e}, a_{j}\right\rangle>0$. Then, $\left\langle\alpha_{e^{\prime}},-\sum_{i \in A} a_{i}-a_{j}\right\rangle=-\left\langle\alpha_{e},-\sum_{i \in A} a_{i}\right\rangle+\left\langle\alpha_{e}, a_{j}\right\rangle$. See Figure 5 . Since $\left\langle\alpha_{e}, a_{j}\right\rangle \neq 0$, the $S^{1}$ weights are not negative multiples, and the $T^{d} \times S^{1}$ weights $\alpha_{e}, \alpha_{e^{\prime}}$ are relatively prime.

Remark 3.3. From the proof of Proposition 3.2, it is evident that the $T^{d}$ action on $M$ is not GKM in the sense of Definition 2.9.

We now give a GKM description of the $T^{d} \times S^{1}$-equivariant cohomology of a hypertoric variety $M$ in the sense of Section 2 . Let $\Gamma=(V, E)$ denote the GKM graph of $M$, and let $H^{*}(\Gamma, \alpha)$ denote its graph cohomology. By Theorem 2.11, Lemma 3.1, and Proposition 3.2, we may conclude that the image of the map

$$
\imath^{*}: H_{T^{d} \times S^{1}}^{*}(M) \longrightarrow H_{T^{d} \times S^{1}}^{*}\left(M^{T^{d} \times S^{1}}\right)
$$

induced by inclusion is an injection, with image $H^{*}(\Gamma, \alpha)$. 


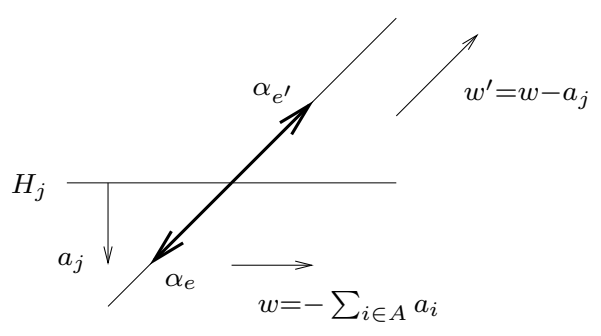

Figure 5: Comparison of the $S^{1}$ weights for two edges on the same hyperplane.

We now have an explicit description of the $T^{d} \times S^{1}$-equivariant cohomology of $M$ as a subring of the sum of polynomial rings $H_{T^{d} \times S^{1}}^{*}\left(M^{T^{d} \times S^{1}} ; \mathbb{Z}\right)$. Another description, in terms of generators and relations, of the $T^{d} \times S^{1}$ equivariant cohomology of $M$ was given in [11]. We will now give a set of ring generators of $H^{*}(\Gamma, \alpha) \cong H_{T^{d} \times S^{1}}^{*}(M ; \mathbb{Z})$ by constructing an isomorphism between the quotient description of the $T^{d} \times S^{1}$-equivariant cohomology given in [11] and the GKM description via $H^{*}(\Gamma, \alpha)$. We first recall the following theorem. The $F_{i}, G_{i}$ are defined in equation (3.2).

Theorem 3.4. $[11,4.4]$ Let $M$ be the hypertoric variety satisfying the hypotheses of Lemma 3.1. Given any minimal set $S \subseteq\{1, \ldots, n\}$ such that $\cap_{i \in S} H_{i}=\emptyset$, let $S=S_{1} \sqcup S_{2}$ be the unique splitting of $S$ such that

$$
\left(\cap_{i \in S_{1}} G_{i}\right) \cap\left(\cap_{j \in S_{2}} F_{j}\right)=\emptyset .
$$

Denote

$$
\mathcal{I}=\left\langle\prod_{i \in S_{1}} u_{i} \times \prod_{j \in S_{2}}\left(x-u_{j}\right) \mid \bigcap_{i \in S} H_{i}=\emptyset\right\rangle .
$$

Then, the $T^{d} \times S^{1}$-equivariant cohomology of $M$ is given by

$$
H_{T^{d} \times S^{1}}^{*}(M ; \mathbb{Z}) \cong \mathbb{Z}\left[u_{1}, \ldots, u_{n}, x\right] / \mathcal{I}
$$

The isomorphism between this quotient description and the GKM description of $H_{T^{d} \times S^{1}}^{*}(M ; \mathbb{Z})$ which we present below is similar in spirit to the isomorphism between the corresponding descriptions for the $T^{d}$-equivariant cohomology ring of the Kähler toric variety $X$. The essential geometric 
insight is to recognize the generators $u_{i}$ as the $T^{d} \times S^{1}$-equivariant Chern classes of certain natural line bundles over $M$.

We first set some notation. Let $v=\cap_{i \in I} H_{i}$ be a vertex. For each such $v$, we define the following subsets of $\{1,2, \ldots, n\}$ :

$$
\begin{aligned}
I_{v} & :=\left\{i \mid v \in H_{i}\right\}, \\
J_{v} & :=\left\{i \mid v \in F_{i}, v \notin H_{i}\right\}, \\
K_{v} & :=\left\{i \mid v \in G_{i}, v \notin H_{i}\right\} .
\end{aligned}
$$

Clearly, $I_{v}=I$, the three sets $I_{v}, J_{v}, K_{v}$ are pairwise disjoint, and the union $I_{v} \cup J_{v} \cup K_{v}=\{1,2, \ldots, n\}$. For $v$ a vertex and $i \in I_{v}$, we define $\eta_{v, i} \in\left(\mathfrak{t}^{d}\right)_{\mathbb{Z}}^{*}$ to be the element satisfying

$$
\left\langle\eta_{v, i}, a_{j}\right\rangle=0, \quad \forall j \in I_{v} \backslash\{i\}, \quad \text { and } \quad\left\langle\eta_{v, i}, a_{i}\right\rangle=1 .
$$

This is well-defined since we assume $\mathcal{H}$ is simple, so the vectors $\left\{a_{i}\right\}_{i \in I_{v}}$ form a $\mathbb{Z}$ basis for $\mathfrak{t}_{\mathbb{Z}}^{d}$.

We now give a GKM description of the $T \times S^{1}$-equivariant cohomology of hypertoric varieties. We specify a $T^{d} \times S^{1}$ weight as a pair

$$
(\alpha, c) \in\left(\mathfrak{t}^{d}\right)_{\mathbb{Z}}^{*} \oplus \mathbb{Z} .
$$

Theorem 3.5. Let $M$ be a hypertoric variety satisfying the hypotheses of Lemma 3.1, $\mathcal{I}$ the ideal given in (3.5), and $H^{*}(\Gamma)$ denote the graph co-

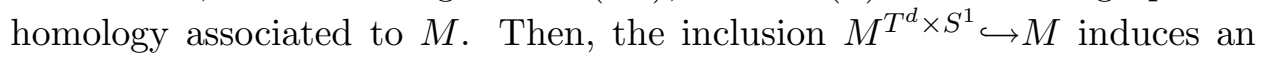
isomorphism

$$
H_{T^{d} \times S^{1}}^{*}(M ; \mathbb{Z}) \cong \mathbb{Z}\left[u_{1}, \ldots, u_{n}, x\right] / \mathcal{I} \cong H^{*}(\Gamma)
$$

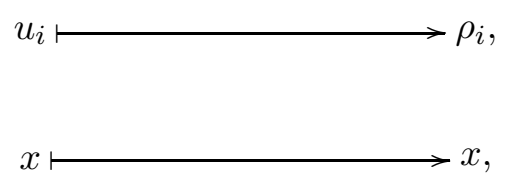

where $\rho_{i}$ is given by

$$
\rho_{i}(v)= \begin{cases}\left(\eta_{v, i},\left\langle\eta_{v, i}, \sum_{j \in K_{v}} a_{j}\right\rangle\right), & \text { if } i \in I_{v} \\ (0,0), & \text { if } i \in J_{v} \\ (0,1), & \text { if } i \in K_{v}\end{cases}
$$


and where $x \in H^{*}(\Gamma)$ denotes the equivariantly constant class corresponding to the integral basis element for $\operatorname{Lie}\left(S^{1}\right)$.

Proof. Let $\left\{h_{i}\right\}_{i=1}^{n}$ be the standard basis of $\left(\mathfrak{t}^{n}\right)_{\mathbb{Z}}^{*}$, and $\tilde{L}_{i}$ be the topologically trivial bundle over $T^{*} \mathbb{C}^{n}$ with $T^{n} \times S^{1}$-equivariant Chern class $h_{i}$. Let $L_{i}$ be the quotient bundle $\left.\tilde{L}_{i}\right|_{\mu_{H K}^{-1}(\alpha, 0)} / T^{k}$. Then, the classes $u_{i}$ are the $T^{d} \times S^{1}$ equivariant Chern classes of $L_{i}$ [11]. In order to compute the images of $u_{i}$ in $H^{*}(\Gamma)$, it suffices to calculate explicitly the $T^{d} \times S^{1}$ action on each fiber $L_{i, p}:=\left.L_{i}\right|_{p}$ for $p \in M^{T^{d} \times S^{1}}$.

Let $v=\cap_{i \in I_{v}} H_{i}$ be the vertex corresponding to the fixed point $p$. Let $\pi: Y=\mu_{H K}^{-1}(\alpha, 0) \rightarrow M$ denote the quotient by $T^{k}$, and let $(z, w) \in Y$ be a preimage of the fixed point $p$. By the moment map conditions and by the definitions of $I_{v}, J_{v}, K_{v}$, we have

$$
\begin{cases}z_{i}=w_{i}=0 & i \in I_{v} \\ z_{i} \neq 0, w_{i}=0 & i \in J_{v} \\ z_{i}=0, w_{i} \neq 0 & i \in K_{v} .\end{cases}
$$

For each $i \in\{1,2, \ldots, n\}$, we wish to compute the restriction of $u_{i}=c_{1}\left(L_{i}\right)$ to the fixed point $p$ corresponding to the vertex $v$. Let $\gamma_{v, i}$ denote the $T^{d}$ weight component of $\left.u_{i}\right|_{p}$. Since the vectors $\left\{a_{j}\right\}_{j \in I_{v}}$ form a $\mathbb{Z}$ basis for $\left(\mathfrak{t}^{d}\right)_{\mathbb{Z}}^{*}$, in order to completely specify $\gamma_{v, i}$, it suffices to compute the pairing $\left\langle\gamma_{v, i}, a_{j}\right\rangle$ for all $j \in I_{v}$. Since we will do our computations on the preimage $\pi^{-1}(p)$, it will be convenient to do computations with $\beta^{*}\left(\gamma_{v, i}\right)$, where $\beta^{*}$ is defined by taking the dual of the exact sequence (3.1). Let $\left\{\epsilon_{j}\right\}$ denote the standard basis for $\mathfrak{t}^{n}$, and let $t_{j}$ denote the elements in the corresponding $S^{1}$ 's in $T^{n}$. Let $((z, w), q)$ denote an element in the total space of the line bundle $\tilde{L}_{i}$ over the point $(z, w)$. For $j \in I_{v}, z_{j}=w_{j}=0$, so the action of $t_{j}$ on $((z, w), q)$ is given by

$$
t_{j} \cdot((z, w), q)= \begin{cases}((z, w), q) & j \neq i \\ \left((z, w), t_{i} q\right) & j=i .\end{cases}
$$

Hence, the $T^{n}$ weight $\beta^{*}\left(\gamma_{v, i}\right)$ satisfies $\left\langle\beta^{*}\left(\gamma_{v, i}\right), \epsilon_{j}\right\rangle=\left\langle\gamma_{v, i}, a_{j}\right\rangle=0$, for all $j \neq i, j \in I_{v}$, as well as $\left\langle\beta^{*}\left(\gamma_{v, i}\right), \epsilon_{i}\right\rangle=\left\langle\gamma_{v, i}, a_{i}\right\rangle=1$. Hence, $\gamma_{v, i}=\eta_{v, i}$, by definition of $\eta_{v, i}$ in (3.7).

We now compute the $S^{1}$ weight component of $\left.u_{i}\right|_{p}$. Recall that the extra $S^{1}$ action on $Y \subseteq T^{*} \mathbb{C}^{n}$ is given by rotating the cotangent direction. So, for an element $s \in S^{1}$,

$$
s \cdot(z, w)=(z, s w)
$$


To compute the $S^{1}$ action on the fiber of $\tilde{L}_{i}$ over $(z, w)$, we must find an element in $T^{k}$ taking $(z, s w)$ back to $(z, w)$. The subtorus $T^{k}$ is defined by the exact sequence (3.1). In particular, an element $\Lambda=\sum_{j=1}^{n} c_{j} \epsilon_{j} \in \mathfrak{t}^{k}$ if and only if $\beta(\Lambda)=\sum_{j=1}^{n} c_{j} a_{j}=0$. Observe that $w_{j} \neq 0$ exactly when $j \in K_{v}$, and that $z_{j} \neq 0$ exactly when $j \in J_{v}$. Hence, the appropriate element in $T^{k}$ will be an exponential of $\Lambda=\sum_{j=1}^{n} c_{j} \epsilon_{j} \in \mathfrak{t}^{k}$ with the conditions $c_{j}=1$ for $j \in K_{v}$ and $c_{j}=0$ for $j \in J_{v}$. ${ }^{2}$ Since the $\left\{a_{j}\right\}_{j \in I_{v}}$ are an integral basis for $\left(\mathfrak{t}^{d}\right)_{\mathbb{Z}}^{*}$, there is a unique integral solution $\left\{m_{j}\right\}_{j \in I_{v}}$ to the equation

$$
\sum_{j \in I_{v}} m_{j} a_{j}+\sum_{j \in K_{v}} a_{j}=0
$$

The $S^{1}$ weight on the fiber of $L_{i}$ is then given by $m_{i}$ for $i \in I_{v}$. Since $\eta_{v, i}$ satisfies the conditions (3.7), the coefficient $m_{i}$ can be computed by the pairing $\left\langle\eta_{v, i},-\sum_{j \in K_{v}} a_{j}\right\rangle$, as desired.

Now, we take the case $i \in J_{v}$. Observe that $\tilde{L}_{i}$ has a $T^{n} \times S^{1}$-equivariant section $\tilde{s}_{i}(z, w)=z_{i}$, which descends to a $T^{d} \times S^{1}$-equivariant section $s_{i}$ of $L_{i}$ with zero-section

$$
Z_{i}:=\left\{[z, w] \in M \mid z_{i}=0\right\} .
$$

This zero-section has (real-)moment image $\mu_{\mathbb{R}}\left(Z_{i}\right)=G_{i}$. For $j \in J_{v}$, the vertex $v$ lies in the interior of $F_{j}$, so the section $s_{i}$ is non-zero at $p$. Hence, the $T^{d} \times S^{1}$ action on the fiber of $L_{j}$ at $p$ is trivial, and the $T^{d} \times S^{1}$ weight $\left.u_{j}\right|_{p}$ is $(0,0)$, as desired.

Finally, consider the case $i \in K_{v}$. We first compute the $T^{d}$ weight component $\gamma_{v, i}$ of $\left.u_{i}\right|_{p}$. By the same argument, as for the case $i \in I_{v}$, it suffices to compute the pairings $\left\langle\beta^{*}\left(\gamma_{v, i}\right), \epsilon_{j}\right\rangle$ for $j \in I_{v}$. This time, since $i \notin I_{v}$, the $T^{d}$ action on the fiber is trivial, and $\left\langle\beta^{*}\left(\gamma_{v, i}\right), \epsilon_{j}\right\rangle=\left\langle\gamma_{v, i}, a_{j}\right\rangle=0$ for all $j \in I_{v}$. Hence, the $T^{d}$ weight component of $\left.u_{i}\right|_{p}$ for $i \in K_{v}$ is 0 , as desired. The $S^{1}$ weight component of $\left.u_{i}\right|_{p}$ is given by $m_{i}$ for $i \in K_{v}$ in the solution (3.8). Hence, the $S^{1}$ weight component is 1 for $i \in K_{v}$, as desired.

Remark 3.6. Interpreted geometrically on the moment map image, Theorem 3.5 states that for a vertex $v$ lying on a hyperplane $H_{i}$, the $T^{d}$ weight component $\eta_{v, i}$ of the restriction $\left.u_{i}\right|_{p}$ is specified by the following conditions:

1. the $T^{d}$ weight $\eta_{v, i}$ lies on the edge $\cap_{j \in I_{v}, j \neq i} H_{j}$; and

\footnotetext{
${ }^{2}$ We take the convention that the standard action of $S^{1}$ on $\mathbb{C}$ is given by $t \cdot z=$ $t^{-1} z$. See $[6$, Appendix A] for an explanation.
} 
2. the $T^{d}$ weight $\eta_{v, i}$ has positive inner product with the inward-pointing normal vector $a_{i}$, so in particular, it always points "towards" $\Delta$.

Remark 3.7. It is possible to prove that the images of the $u_{i}$ in $H_{T \times S^{1}}^{*}\left(M^{T \times S^{1}}\right)$ given in Theorem 3.5 do indeed satisfy the GKM conditions. The proof is rather tedious and we do not include it here.

To illustrate Theorem 3.5, we consider the hypertoric varieties determined by the hyperplane arrangements in Figure 6 .

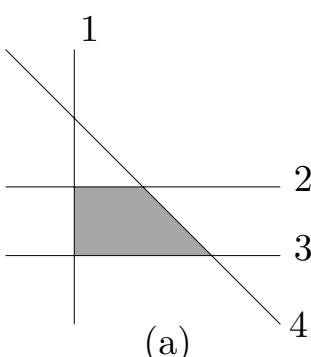

(a)

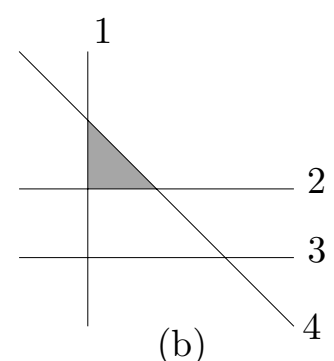

(b)

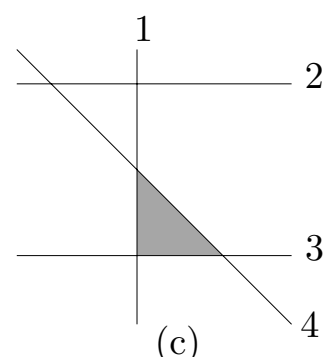

(c)

Figure 6: Three different hypertoric varieties.

Example 3.8. Let $M_{a}$ denote the hypertoric variety specified by the hyperplane arrangement in Figure 6(a). In [11], the equivariant cohomology $H_{T^{d} \times S^{1}}^{*}\left(M_{a} ; \mathbb{Z}\right)$ is computed to be

$$
H_{T^{d} \times S^{1}}^{*}\left(M_{a} ; \mathbb{Z}\right)=\mathbb{Z}\left[u_{1}, \ldots, u_{4}, x\right] /\left\langle u_{2} u_{3}, u_{1}\left(x-u_{2}\right) u_{4}, u_{1} u_{3} u_{4}\right\rangle,
$$

where the $u_{i}$ are Euler classes of $T^{d} \times S^{1}$-equivariant line bundles $L_{i}$ over $M_{a}$.

The images of the $u_{i}$ are given in Figure 7. We choose an integral basis $\left\{e_{1}, e_{2}\right\}$ for $\mathfrak{t}^{d}=\mathfrak{t}^{2}$ as shown in the Figure, and we denote the integral basis element for $\operatorname{Lie}\left(S^{1}\right)$ by $x$. The equivariantly constant class $x$ maps to the GKM class with weight $x$ at each point.

Example 3.9. Now, let $M_{b}$ denote the hypertoric variety given by the arrangement in Figure 6(b). The equivariant cohomology is computed in [11] to be

$$
H_{T^{d} \times S^{1}}^{*}\left(M_{b} ; \mathbb{Z}\right)=\mathbb{Z}\left[u_{1}, \ldots, u_{4}, x\right] /\left\langle\left(x-u_{2}\right) u_{3}, u_{1} u_{2} u_{4}, u_{1} u_{3} u_{4}\right\rangle .
$$

We give the GKM descriptions of the ring generators $u_{i}$ in Figure 8 below. 

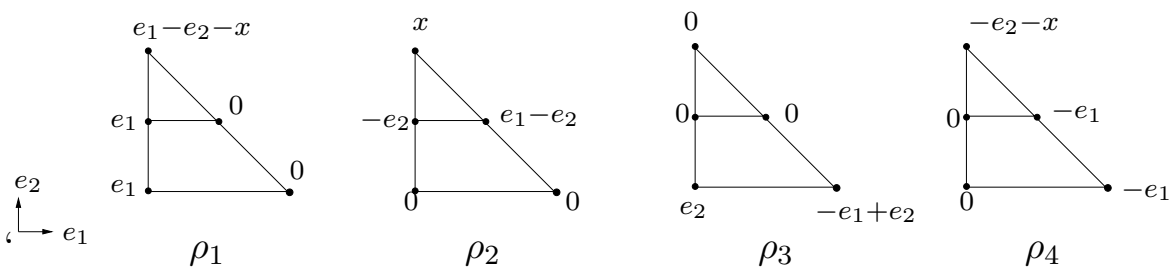

Figure 7: Some ring generators for $H_{T^{d} \times S^{1}}^{*}\left(M_{a} ; \mathbb{Z}\right)$. The plane of the figure is spanned by the two vectors $e_{1}$ and $e_{2}$. The third axis $x$ points out of the page.

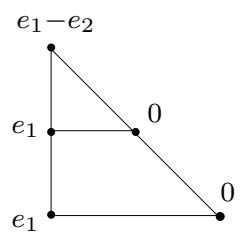

$\rho_{1}$

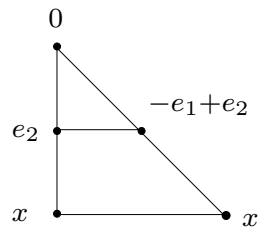

$\rho_{2}$

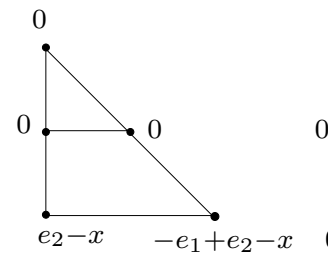

$\rho_{3}$

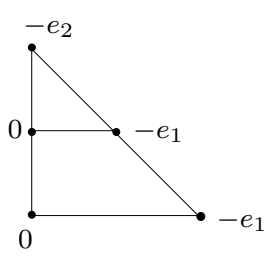

$\rho_{4}$

Figure 8: Some ring generators for $H_{T^{d} \times S^{1}}^{*}\left(M_{b} ; \mathbb{Z}\right)$.

Example 3.10. Let $M_{c}$ be the hypertoric variety given by the arrangement in Figure 6(c). The equivariant cohomology is computed in [11] to be

$$
H_{T^{d} \times S^{1}}^{*}\left(M_{c} ; \mathbb{Z}\right)=\mathbb{Z}\left[u_{1}, \ldots, u_{4}, x\right] /\left\langle u_{2} u_{3},\left(x-u_{1}\right) u_{2}\left(x-u_{4}\right), u_{1} u_{3} u_{4}\right\rangle .
$$

We give the GKM image of the $u_{i}$ in Figure 9 below.

We end this section with a discussion of the $T^{d}$ action on a hypertoric variety, considered as a GKM action. We have already noted in Remark 3.3 that the $T^{d}$ action on $M$ does not satisfy the GKM hypotheses in the sense of Definition 2.9. It does satisfy the more general GKM conditions considered in [10], in which the theory is developed in the language of cell complexes with a compatible $T$ action. Thus, by $\left[10\right.$, Theorem 3.4], $H_{T^{d}}^{*}(M ; \mathbb{Z})$ does admit a GKM description in $H_{T^{d}}^{*}\left(M^{T^{d}} ; \mathbb{Z}\right)$. We will now exploit our knowledge of the GKM description of $H_{T^{d} \times S^{1}}^{*}(M ; \mathbb{Z})$ to give an explicit list of ring generators for $H_{T^{d}}^{*}(M ; \mathbb{Z})$, described as elements of $H_{T^{d}}^{*}\left(M^{T^{d}} ; \mathbb{Z}\right)$.

Remark 3.11. Note that the techniques in [10] do not in general yield ring 


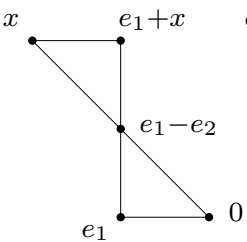

$\rho_{1}$

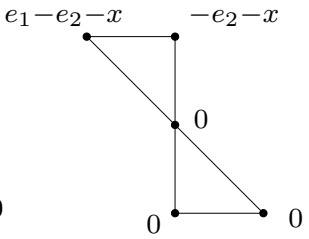

$\rho_{2}$

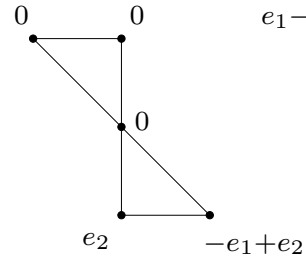

$\rho_{3}$

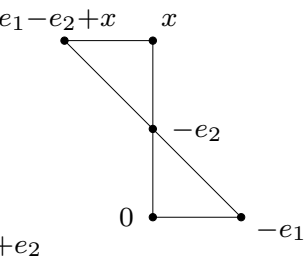

$\rho_{4}$

Figure 9: Some ring generators for $H_{T^{d} \times S^{1}}^{*}\left(M_{c} ; \mathbb{Z}\right)$.

generators for the $T$-equivariant cohomology, so this is a new result from our explicit analysis of $M$ as a $T^{d} \times S^{1}$ space.

We will obtain GKM ring generators for $H_{T^{d}}^{*}(M ; \mathbb{Z})$ by "GKM in stages." First, recall that in the Borel construction $M \times_{T^{d}} E T^{d}$ for $H_{T^{d}}^{*}(M ; \mathbb{Z})$, we may use instead of $E T^{d}$ any contractible space on which $T^{d}$ acts freely. In particular, we may use $E\left(T^{d} \times S^{1}\right)$. Hence, there is a natural map

$$
H_{T^{d} \times S^{1}}^{*}(M ; \mathbb{Z}) \rightarrow H_{T^{d}}^{*}(M ; \mathbb{Z})
$$

induced by the inclusion

$$
M \times_{T^{d}} E\left(T^{d} \times S^{1}\right) \hookrightarrow M \times_{T^{d} \times S^{1}} E\left(T^{d} \times S^{1}\right) .
$$

In our situation, we have in addition that $M^{T^{d}}=M^{T^{d} \times S^{1}}$, so we obtain a commutative diagram

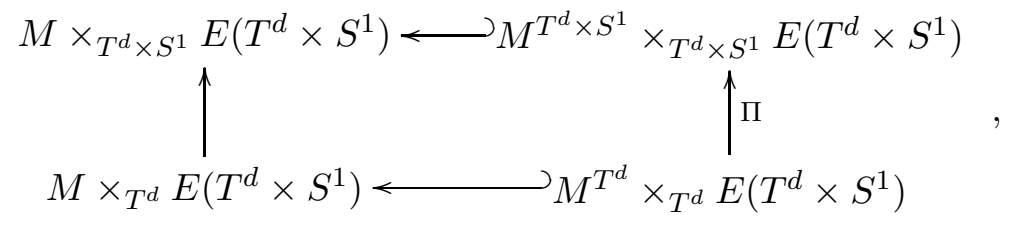

where $\Pi$ uses the equality $M^{T^{d}}=M^{T^{d} \times S^{1}}$. We then have a diagram on equivariant cohomology

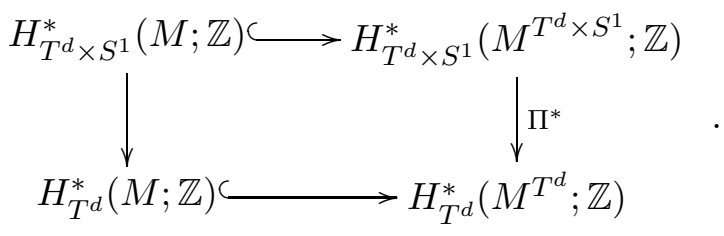


Since the left vertical arrow is a surjection by formality of $H_{T^{d} \times S^{1}}^{*}(M ; \mathbb{Z})$ over $H_{S^{1}}^{*}(p t ; \mathbb{Z})[11]$, the right vertical arrow $\Pi^{*}$ also gives a surjection on the images. Moreover, since the images of the $u_{i}$ generate $H_{T^{d}}^{*}(M)$, in order to give generators for the GKM description of $H_{T^{d}}^{*}(M ; \mathbb{Z})$ in $H_{T^{d}}^{*}\left(M^{T^{d}} ; \mathbb{Z}\right)$, it suffices to compute $\Pi^{*}\left(\rho_{i}\right)$, where the $\rho_{i}$ are given in Theorem 3.5. Note that the map $\Pi^{*}$ is the map that sends $x$ to 0 . We end the section with an example of an explicit computation.

Example 3.12. A set of generators of $H_{T^{d}}^{*}(M ; \mathbb{Z})$, considered in $H_{T^{d}}^{*}\left(M^{T^{d}} ; \mathbb{Z}\right)$, are shown in Figure 10 below. They are the $\pi^{*}\left(\rho_{i}\right)$ for the $\rho_{i}$ given in Example 3.8.
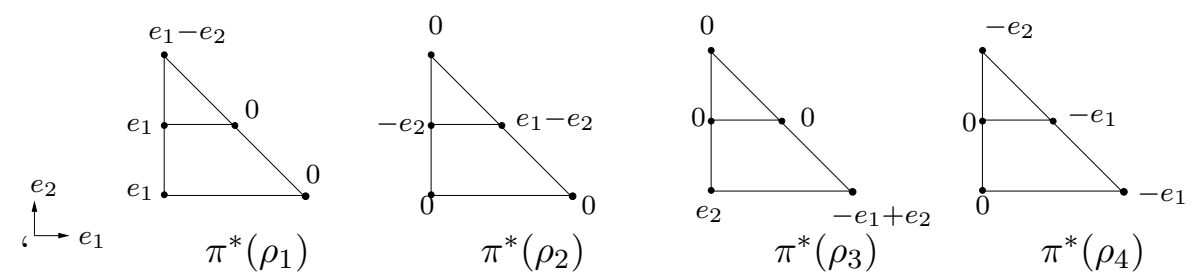

Figure 10: Ring generators for $H_{T^{d}}^{*}\left(M_{a} ; \mathbb{Z}\right)$.

\section{The real locus.}

We now consider the situation in which we have, in addition to a Hamiltonian $T$-action on $M$, an antisymplectic involution $\sigma$ on $M$ which anticommutes with the action of $T$, i.e.

$$
\sigma(t x)=t^{-1} \sigma(x), \quad \forall x \in M, \forall t \in T .
$$

The $\sigma$-fixed points $Q:=M^{\sigma}$ in $M$ is a real $n$-dimensional Lagrangian submanifold of $M$, which we call the real locus of $M$. The real locus $Q$ and its properties (e.g. its image under the $T$ moment map, and its equivariant cohomology) have been extensively studied; see [5], [17], [4], [18]. Most of the known results use the assumption, in addition to certain technical conditions about the $T$ action, that $M$ (and therefore $Q$ ) is compact. We will show in this section that many known results generalize to the situation in which $M$ is not necessarily compact, but a component of the moment map is proper and bounded below. Again, our motivating example is the 
hypertoric variety with its $T \times S^{1}$ action, which will be discussed in detail in Section 5.

We begin our story with an extension of a theorem which states that the image of the real locus under the $T$ moment map coincides with that of the whole manifold $M$, i.e. $\mu(Q)=\mu(M)$. When the manifold $M$ is compact, this result is due to Duistermaat [5].

Proposition 4.1. Let $(M, \omega)$ be a symplectic manifold with a Hamiltonian $T$ action, $T$ moment map $\mu$, and $\sigma$ an antisymplectic involution satisfying (4.1). Denote by $Q$ the real locus of $M$, i.e. $Q:=M^{\sigma}$. Suppose that there is a component of the moment map $\mu$ which is proper and bounded below. Then, $\mu(Q)=\mu(M)$.

Proof. Let $\mu^{\xi}$ be a component of a moment map for $M$ which is proper and bounded below. We may assume $\xi$ is rational. Denote by $S_{\xi}^{1}$ the subtorus in $T$ generated by $\xi$. Since $\sigma(t x)=t^{-1} \sigma(x)$ for all $x \in M, t \in T$, we may assume that $\mu^{\xi}(\sigma(x))=\mu^{\xi}(x)$, for any $x \in M[17,2.2]$. Without loss of generality, we assume 0 is the minimum value of $\mu^{\xi}$ on $M$. Let $\bar{M}_{s}$ be the symplectic cut space of $M$ at the value $s>0$ with respect to the action of $S_{\xi}^{1}$. $\bar{M}_{c+s}$. Then, $\bar{M}_{c+s}$ is equipped with an antisymplectic involution $\bar{\sigma}_{c+s}$, descending from the involution $\tilde{\sigma}(m, z)=(\sigma(m), \bar{z})$ on $M \times \mathbb{C}$, as well as a Hamiltonian $T$ action, descending from the action of $T$ on the first factor. These obey the relation (4.1). Denote by $\bar{\mu}$ the $T$ moment map on $\bar{M}_{s}$, and let $\bar{Q}_{s}:=\left(\bar{M}_{s}\right)^{\bar{\sigma}_{c+s}}$ be its real locus. Since $\mu^{\xi}$ is proper, the symplectic cut space $\bar{M}_{s}$ is compact. Thus, Duistermaat's theorem applies, and $\bar{\mu}\left(\bar{M}_{s}\right)=\bar{\mu}\left(\bar{Q}_{s}\right)$. On the other hand, the symplectic cut space $\bar{M}_{s}$ contains as an open subset the preimage $\left(\mu^{\xi}\right)^{-1}((-\infty, c+s)) \subset M$ in the original manifold, and on this open piece, the involution $\bar{\sigma}_{c+s}$, the Hamiltonian $T$ action, and the $T$ moment map $\mu$ all agree with those just defined on $\bar{M}_{s}$. Since $s$ was arbitrary, we may conclude that $\mu(M)=\mu(Q)$.

Remark 4.2. Note that for the above proposition, we do not need to assume that the $T$ action is GKM. We only need that the $T$ moment map is proper and a component is bounded below.

We now turn our attention to the mod 2 GKM theory for the real locus $Q$. Since the $T$ action on $M$ anticommutes with $\sigma$, there is a subgroup $\{ \pm 1\}^{n}=\left(\mathbb{Z}_{2}\right)^{n} \subseteq T^{n}$, denoted $T_{\mathbb{R}}$, which preserves $Q$. Thus, we can speak of the $T_{\mathbb{R}}$-equivariant cohomology of $Q$, and we will show that under certain 
conditions, we have an isomorphism of graded rings

$$
H_{T}^{2 *}\left(M ; \mathbb{Z}_{2}\right) \cong H_{T_{\mathbb{R}}}^{*}\left(Q ; \mathbb{Z}_{2}\right)
$$

that halves the grading. (For the compact case, see $[4,18]$.)

Henceforth, we assume that the $T$ action on $M$ is GKM. In order to get the isomorphism of graded rings described above, we will need additional assumptions on the $T$-isotropy weights at the fixed points. We first set up the notation. The $\bmod 2$ reduction of a weight $\alpha \in \mathfrak{t}_{\mathbb{Z}}^{*}$ in the weight lattice of $T$ is defined to be its image in $\mathfrak{t}_{\mathbb{Z}}^{*} / 2 \mathfrak{t}_{\mathbb{Z}}^{*}$. We will denote by $\bar{\alpha}_{p, i}$ the $\bmod 2$ reduction of a $T$ weight $\alpha_{p, i}$ at a $T$-fixed point $p$.

Definition 4.3. Let $M$ be a manifold equipped with a $T$ action. Then, the action is $\bmod 2 G K M$ if it is GKM and, for every $p \in M^{T}$, the $\bmod 2$ reduced weights $\left\{\bar{\alpha}_{p, i}\right\}_{i=1}^{n}$ are all distinct and non-zero.

Remark 4.4. In [18], the term $\mathbb{Z}_{2}$ pure is used; this is equivalent to $\bmod 2$ GKM.

In Section 2, we have already shown that the GKM theorem holds for non-compact GKM actions if certain conditions hold on the moment map. In order to show the isomorphism of the two cohomology rings $H_{T}^{2 *}\left(M ; \mathbb{Z}_{2}\right)$ and $H_{T_{\mathbb{R}}}^{*}\left(Q ; \mathbb{Z}_{2}\right)$, we need now to show that the analogous results hold for a non-compact real locus $Q$ in the case where the $T$ action is also mod 2 GKM. We will use the Morse theory of the restricted moment map $\left.\mu\right|_{Q}$ on $Q$. Let $P$ be the set

$$
P:=\left\{x \in Q \mid \operatorname{codim}_{\mathbb{Z}_{2}}\left(\operatorname{Stab}_{T_{\mathbb{R}}}(x)\right)=1\right\} .
$$

As before, we define the one-skeleton $\bar{P}$ of the $T_{\mathbb{R}}$ action on $Q$ to be the closure of $P$.

We first claim that when the $T$ action is mod 2 GKM, then

$$
M^{T}=Q^{T_{\mathbb{R}}}
$$

and

$$
\bar{P}=\bar{N} \cap Q
$$

Thus, the combinatorics of the mod 2 one-skeleton $\bar{P}$ for the $T_{\mathbb{R}}$ action on $Q$ is the same as that of the one-skeleton $\bar{N}$. We give below a sketch of a proof of the equality (4.2) because there is a gap in its proof in [4, Theorem 5.2]. We outline the argument in [18, Proposition 5.1.6], and we include this here because Schmid's thesis is not available in print. 
Proposition 4.5. Let a torus $T$ act on a symplectic manifold $M$ with moment map $\mu: M \rightarrow \mathfrak{t}^{*}$ that is proper and bounded below in some generic component. Suppose further that $M$ is equipped with an antisymplectic involution $\sigma$ that anticommutes with the $T$ action, and that the $T$ action is $\bmod 2$ GKM. Then, $M^{T}=Q^{T_{\mathbb{R}}}$.

Proof. We must first show that $Q^{T_{\mathbb{R}}} \subseteq M^{T}$. This is what is shown in the proof of [4, Theorem 5.2]. The proof of this uses the fact that the isotropy weights have non-zero mod 2 reductions. The gap in the proof is that the reverse inclusion $M^{T} \subseteq Q^{T_{\mathbb{R}}}$ is not addressed. We complete that now.

It suffices to show that $M^{T} \subseteq Q$. We first show that there is at least one $T$-fixed point in $Q$. Let $\mu^{\xi}$ be a generic component of $\mu$ that is proper and bounded below. Since the fixed points are isolated, there is exactly one fixed point $p \in M^{T}$ mapping to the minimum value of $\mu^{\xi}$. By Proposition 4.1, we have $\mu^{\xi}(Q)=\mu^{\xi}(M)$, and so, we must have $p \in Q$.

Now, we show that every $T$-fixed point is in $Q$. Let $(p, q)$ be an edge in $\Gamma$, corresponding to an embedded $\mathbb{C} P^{1}$, where $p$ is a vertex known to be in $Q$. This $\mathbb{C} P^{1}$ is fixed by a codimension 1 subtorus $T^{\prime} \leq T$. It is a connected component of $M^{T^{\prime}}$, and $M^{T^{\prime}}$ is preserved by $\sigma$. As $p \in Q$, this copy of $\mathbb{C} P^{1}$ must itself be preserved by $\sigma$. Applying Proposition 4.1 to this $\mathbb{C} P^{1}$ allows us to conclude that $q$ is also an element of $Q$. Finally, because the one-skeleton is connected, it follows that every $T$-fixed point is in $Q$. This completes the proof.

Remark 4.6. In the compact setting, (4.3) is proved in [4, Theorem 5.2] and in [18, Proposition 5.1.5]. In the non-compact setting, this follows by a cutting argument similar to that given in the proof of Proposition 4.1.

We assume from now on that the $T$ action on our manifold $M$ is $\bmod 2$ GKM. In this situation, the moment map $\mu$, restricted to $Q$, also behaves quite nicely. By our assumptions on $M$, there is a component of the moment map $\mu^{\xi}$ which is proper and bounded below, and (since the action is GKM) is a Morse function on $M$. In [5], Duistermaat showed that the restriction of $\mu^{\xi}$ to $Q$ is also a Morse function on $Q$, and has critical points exactly $M^{T} \cap Q$ when $M$ is compact. In our situation, his argument goes through to show that components of $\mu$ are again Morse functions on $Q$. Thus, this allows us to compute the equivariant cohomology $H_{T_{\mathbb{R}}}^{*}\left(Q ; \mathbb{Z}_{2}\right)$ using an argument very similar to that given in Section 2.

The statement of the analogous mod 2 GKM theorem for real loci will require the definition of a mod 2 GKM graph $\Gamma_{\mathbb{R}}$. The vertices $V_{\mathbb{R}}$ of $\Gamma_{\mathbb{R}}$ 
are the fixed points $Q^{T_{\mathbb{R}}}$. The edges are given by the components of the one-skeleton $P$ the closure of which is an $S^{1}$, and they connect the two fixed points in $Q^{T_{\mathbb{R}}}$ contained in this $S^{1}$. To each edge $e$, we associate a weight $\bar{\alpha}_{e}$ of the $T^{\mathbb{R}}$ action on this $S^{1}$. We then define the graph cohomology $H^{*}\left(\Gamma_{\mathbb{R}}, \alpha_{\mathbb{R}}\right)$ to be

$$
\begin{aligned}
H^{*}\left(\Gamma_{\mathbb{R}}, \alpha_{\mathbb{R}}\right) & =\left\{f: V \rightarrow H_{T_{\mathbb{R}}}^{*}\left(p t ; \mathbb{Z}_{2}\right) \mid \begin{array}{c}
f(p)-f(q) \equiv 0\left(\bmod \bar{\alpha}_{e}\right) \\
\text { for every edge } e=(p, q)
\end{array}\right\} \\
& \subseteq H_{T}^{*}\left(V ; \mathbb{Z}_{2}\right) .
\end{aligned}
$$

Note that for $T_{\mathbb{R}}=\left(\mathbb{Z}_{2}\right)^{d}$, the $T_{\mathbb{R}}$-equivariant cohomology $H_{T_{\mathbb{R}}}^{*}\left(p t ; \mathbb{Z}_{2}\right)$ is a polynomial ring over $\mathbb{Z}_{2}$ with $d$ generators, where the generators are degree 1 instead of degree 2 .

We will need the following mod 2 version of the Atiyah-Bott lemma. For a proof, see [2, Proposition 5.3.7] or [7, Lemma 2.3].

Lemma 4.7 (Atiyah-Bott). Let $\mathcal{E} \rightarrow B$ be a real rank $\ell$ vector bundle over a compact manifold $B$. Let $T_{\mathbb{R}}$ be the group $T_{\mathbb{R}}=\left(\mathbb{Z}_{2}\right)^{d}$. Suppose that $T_{\mathbb{R}}$ acts on $\mathcal{E}$ with fixed point set precisely $B$. Choose a $T_{\mathbb{R}}$-invariant Riemannian metric on $\mathcal{E}$, and let $D$ and $S$ be the corresponding disk and sphere bundles, respectively, of $\mathcal{E}$. Then, the long exact sequence of the pair $(D, S)$ splits into short exact sequences

$$
0 \longrightarrow H_{T_{\mathbb{R}}}^{*}\left(D, S ; \mathbb{Z}_{2}\right) \longrightarrow H_{T_{\mathbb{R}}}^{*}\left(D ; \mathbb{Z}_{2}\right) \longrightarrow H_{T_{\mathbb{R}}}^{*}\left(S ; \mathbb{Z}_{2}\right) \longrightarrow 0
$$

We now prove the real locus version of Theorem 2.11.

Theorem 4.8. Let $M$ be a Hamiltonian $T$ space with moment map $\mu$. Assume that there is a generic direction $\xi$ such that $\mu^{\xi}$ is proper and bounded below. Suppose further that $M$ is equipped with an antisymplectic involution $\sigma$ that anticommutes with the $T$ action, and that the $T$ action is $\bmod$ 2 GKM. Let $Q$, denote the real locus. Then, the inclusion $Q^{T_{\mathbb{R}}} Q$ induces an injection

$$
H_{T_{\mathbb{R}}}^{*}\left(Q ; \mathbb{Z}_{2}\right) \hookrightarrow H_{T_{\mathbb{R}}}^{*}\left(Q^{T_{\mathbb{R}}} ; \mathbb{Z}_{2}\right),
$$

in equivariant cohomology, and the image is precisely $H^{*}\left(\Gamma_{\mathbb{R}}, \bar{\alpha}\right)$.

Proof. The outline of the proof is the same as that given in Section 2. We will only mention the relevant steps where some additional argument is necessary.

We begin with the mod 2 version of Proposition 2.4. To get the statement, we replace $M$ with the real locus $Q$, the Morse-Bott function $\mu^{\xi}$ with 
$g:=\left.\mu^{\xi}\right|_{Q}, T$ with $T_{\mathbb{R}}$, and Euler classes with Stiefel-Whitney classes. This follows from Lemma 4.7 by the same argument as in Proposition 2.4. Thus, the restriction from $H_{T_{\mathbb{R}}}^{*}\left(Q_{c}^{+} ; \mathbb{Z}_{2}\right)$ to $H_{T_{\mathbb{R}}}^{*}\left(Q_{c}^{-} ; \mathbb{Z}\right)$ induces an isomorphism from the kernel of $k^{*}$ to those classes in $H_{T_{\mathbb{R}}}^{*}\left(p ; \mathbb{Z}_{2}\right)$ which are multiples of $\kappa_{p}$, the equivariant Stiefel-Whitney class of the negative normal bundle to $p$ with respect to $g$. We are assuming the action is $\bmod 2$ GKM, so the fixed point components are isolated points. The mod 2 version of the injectivity theorem, Theorem 2.6, now follows by the same argument, using the $T_{\mathbb{R}}$-equivariant Thom isomorphism theorem with $\mathbb{Z}_{2}$ coefficients.

Before proceeding to the mod 2 analogue of Theorem 2.7, we first take a moment to analyze the $T_{\mathbb{R}}$-isotropy weights at the fixed points $Q^{T_{\mathbb{R}}}$, which are the critical points of $g$. Let $p$ be a fixed point in $M^{T}=Q^{T_{\mathbb{R}}}$. There exists a neighborhood of $p$ equivariantly symplectomorphic to a neighborhood of 0 in $T_{p} M$ with the symplectic form $\omega_{p}$. Moreover, since $p \in Q$, the involution $\sigma$ acts on $T_{p} M$, anticommuting with the action of $T$. The local normal form theorem in [17, Theorem 7.1] implies that there exists a $T$-invariant, $\sigma$-anti-invariant compatible complex structure on $T_{p} M$ making it a complex vector space, and as a $T_{\mathbb{R}}$ module, $T_{p} M$ is canonically isomorphic to the complexification of $\left(T_{p} M\right)^{\sigma}$. More specifically, we have local coordinates such that a neighborhood of $p$ in $M$ is of the form $\oplus_{i=1}^{n} \mathbb{C}_{\alpha_{i, p}}$, where the $\alpha_{i, p}$ are the $T$-isotropy weights at $p$, and $\sigma$ is given by complex conjugation on each factor. Then, a neighborhood of $p$ in $Q$ in these coordinates is of

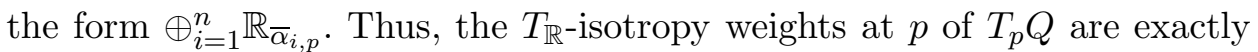
the mod 2 reductions of the $T$-isotropy weights $\left\{\alpha_{i, p}\right\}$. In particular, the $T_{\mathbb{R}}$-equivariant Stiefel-Whitney class of the negative normal bundle in $Q$ with respect to $g$ is given by the product of the $T_{\mathbb{R}}$ weights $\bar{\alpha}_{i, p}$ for which $\left\langle\alpha_{i, p}, \xi\right\rangle<0$. Since by assumption, all $T_{\mathbb{R}}$ weights are non-zero, the product is also non-zero in $H_{T_{\mathbb{R}}}^{*}\left(p ; \mathbb{Z}_{2}\right)$, and therefore, not a zero divisor. Finally, we note that two elements $\bar{\alpha}, \overline{\alpha^{\prime}}$ in $H_{T_{\mathbb{R}}}^{*}\left(p ; \mathbb{Z}_{2}\right)$ are relatively prime if they are non-zero and distinct.

Using the above observations, the mod 2 version of Theorem 2.7 follows from the same argument as in Section 2.

In order to compute $H_{T_{\mathbb{R}}}^{*}\left(Q ; \mathbb{Z}_{2}\right)$, it now suffices to compute the image of $H_{T_{\mathbb{R}}}^{*}\left(\bar{P} ; \mathbb{Z}_{2}\right)$ in the ring $H_{T_{\mathbb{R}}}^{*}\left(Q^{T_{\mathbb{R}}} ; \mathbb{Z}_{2}\right)$. Finally, to observe the isomorphism between the graded rings $H_{T}^{2 *}\left(M ; \mathbb{Z}_{2}\right)$ and $H_{T_{\mathbb{R}}}^{*}\left(Q ; \mathbb{Z}_{2}\right)$, it suffices to compare the relevant graphs. Note that since $M^{T}=Q^{T_{\mathbb{R}}}$, the vertices of the graphs $\Gamma$ and $\Gamma_{\mathbb{R}}$ are the same, and because $\bar{P}=\bar{N} \cap Q$, the graphs are the same. Moreover, by the argument given in the proof of Theorem 4.8, the 
images mod 2 of the isotropy weights $\alpha_{e}$ on the edges of $\Gamma$ are exactly the $T_{\mathbb{R}}$-isotropy weights on the edges of $\Gamma_{\mathbb{R}}$. Thus, the combinatorial data specified by the GKM and mod 2 GKM graphs are identical, and the following corollary is immediate.

Corollary 4.9. Let $M$ be a Hamiltonian $T$ space with moment map $\mu$. Assume that there is a generic direction $\xi$ such that $\mu^{\xi}$ is proper and bounded. Suppose further that $M$ is equipped with an antisymplectic involution $\sigma$ that anticommutes with the $T$ action, and that the $T$ action is mod 2 GKM. Let $Q$ denote the real locus. Then, there is an isomorphism

$$
H_{T}^{2 *}\left(M ; \mathbb{Z}_{2}\right) \cong H_{T_{\mathbb{R}}}^{*}\left(Q ; \mathbb{Z}_{2}\right)
$$

that halves degrees.

\section{Examples: real loci of hypertoric varieties.}

The hypertoric varieties in Section 3 have a natural antisymplectic involution $\sigma$, induced from the antisymplectic involution on $T^{*} \mathbb{C}^{n}$ given by $(z, w) \mapsto$ $(\bar{z}, \bar{w})$. We now analyze the topology of the real locus of $M$ using techniques of the previous section.

Let $M$ be a hypertoric variety specified by a hyperplane arrangement $\mathcal{H}$ and parameter $\alpha$. Then, the real locus $Q$ of $M$ is the set

$$
Q:=M^{\sigma}=\{[z, w] \in M \mid z, w \in \mathbb{R}\} .
$$

Since the hypertoric variety has an action of $T^{d} \times S^{1}$, the group acting on $Q$ is now $T_{\mathbb{R}}=T_{\mathbb{R}}^{d} \times \mathbb{Z}_{2}$.

In this situation, the isomorphism between the $T_{\mathbb{R}} \times \mathbb{Z}_{2}$-equivariant cohomology of $Q$ and the $T \times S^{1}$-equivariant cohomology of $M$ (both with $\mathbb{Z}_{2}$ coefficients) can be explicitly described in terms of the line bundles $L_{i}$ over $M$.

Proposition 5.1. Let $M$ be a hypertoric variety specified by $\mathcal{H}$ and $\alpha$. There are antisymplectic involutions $\sigma_{i}$ on the total spaces of the $L_{i}$, extending the natural involution $\sigma$ on $Q$, so that the fixed point sets $L_{i}^{\sigma}$ are real vector bundles over $Q$. Moreover, under the isomorphism in Corollary 4.9, the Chern class $u_{i}$ of $L_{i}$ is mapped to the Stiefel-Whitney class $\kappa_{i}$ of the real bundle $L_{i}^{\sigma}$.

Proof. For each line bundle $\tilde{L}_{i}$ over $T^{*} \mathbb{C}^{n}$, define an involution $\tilde{\sigma}_{i}$ in coordinates by

$$
\tilde{\sigma}_{i} \cdot(z, w, q):=(\bar{z}, \bar{w}, \bar{q})
$$


This is a lift of the standard antisymplectic involution on $T^{*} \mathbb{C}^{n}$. The $\tilde{\sigma}_{i^{-}}$

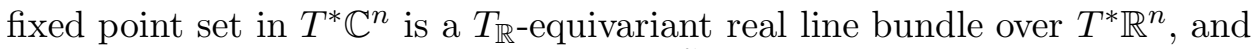
its complexification is the restriction of $\tilde{L}_{i}$ to $T^{*} \mathbb{R}^{n}$. Since the $\tilde{\sigma}_{i}$ are anti$T$-equivariant, they descend to antisymplectic involutions $\sigma_{i}$ on the $L_{i}$ on $M$. The $\sigma_{i}$-fixed point sets are $T_{\mathbb{R}}$-equivariant real line bundles over the real locus $Q$, and their complexifications are $\left.L_{i}\right|_{Q}$.

Since the complexification of $\left(L_{i}\right)^{\sigma_{i}}$ is isomorphic as a real bundle to $L_{i}^{\sigma_{i}} \oplus L_{i}^{\sigma_{i}}$, we have $\kappa_{2}\left(L_{i}^{\sigma_{i}}\right)=\kappa_{1}\left(L_{i}^{\sigma_{i}}\right)^{2}$. Under the natural homomorphism $H^{2}(Q ; \mathbb{Z}) \rightarrow H^{2}\left(Q ; \mathbb{Z}_{2}\right)$, the image of the Chern class of a complex line bundle is the second Stiefel-Whitney class, so we may conclude that the isomorphism between $H_{T}^{*}(M ; \mathbb{Z})$ and $H_{T_{\mathbb{R}}}^{*}\left(Q ; \mathbb{Z}_{2}\right)$ takes the $\bmod 2$ Chern class of $\left.L_{i}\right|_{Q}$ to the Stiefel-Whitney class $\kappa_{1}$ of $L_{i}^{\sigma_{i}}$.

Remark 5.2. The presentation of the $T^{d} \times S^{1}$-equivariant cohomologies given in Examples 3.8, 3.9, and 3.10 is therefore, identical to that of the $T_{\mathbb{R}}^{d} \times$ $\mathbb{Z}_{2}$-equivariant cohomologies of their real loci, where we use $\mathbb{Z}_{2}$ coefficients and divide all degrees of the classes in half.

Remark 5.3. The techniques developed in Section 4, and this description of $H_{T_{\mathbb{R}}^{d} \times \mathbb{Z}_{2}}^{*}\left(Q ; \mathbb{Z}_{2}\right)$ is used in [11] to compute a deformation of the OrlikSolomon algebra of a smooth real hyperplane arrangement, depending nontrivially on the affine structure of the arrangement.

\section{Acknowledgements.}

We would like to take this opportunity to thank the Mathematical Sciences Research Institute for their support while we wrote part of this paper. We would also like to thank the referee for useful comments.

\section{References.}

[1] M. Atiyah and R. Bott. The Yang-Mills equations over Riemann surfaces. Phil. Trans. R. Soc. Lond., 308:523-615, 1982.

[2] C. Allday and V. Puppe. Cohomological methods in transformation groups, volume 32 of Cambridge Studies in Advanced Mathematics. Cambridge University Press, Cambridge, 1993.

[3] R. Bielawski and A. Dancer. The geometry and topology of toric hyperkähler manifolds. Comm. Anal. Geom., 8:727-760, 2000. 
[4] D. Biss, V. Guillemin, and T. Holm. The mod 2 cohomology of fixed point sets of antisymplectic involutions. math.SG/0107151, 2001.

[5] H. Duistermaat. Convexity and tightness for restrictions of Hamiltonian functions to fixed point sets of anti-symplectic involutions. Trans. AMS, 275(1):417-429, 1983.

[6] V. Ginzburg, V. Guillemin, and Y. Karshon. Moment maps, cobordisms, and Hamiltonian group actions. Number 98 in Mathematical Surveys and Monographs. American Mathematical Society, 2002.

[7] R. Goldin and T. Holm. Real loci of symplectic reductions. Trans. $A M S$, to appear 2004.

[8] M. Goresky, R. Kottwitz, and R. MacPherson. Equivariant cohomology, Koszul duality, and the localization theorem. Invent. Math., 131:25-83, 1998.

[9] V. Guillemin and C. Zara. 1-skeleta, Betti numbers, and equivariant cohomology. Duke Math. J., 107(2):283-349, 2001.

[10] M. Harada, A. Henriques, and T. Holm. T-equivariant cohomology of cell complexes and the case of infinite Grassmannians. math.DG/0402079, 2004.

[11] M. Harada and N. Proudfoot. Properties of the residual circle action on a hypertoric variety. math.DG/0207012, 2002.

[12] T. Hausel and B. Sturmfels. Toric hyperkähler varieties. Doc. Math., $7: 495-534,2002$.

[13] F. Kirwan. Cohomology of quotients in symplectic and algebraic geometry, volume 31 of Mathematical Notes. Princeton University Press, Oxford, 1984.

[14] H. Konno. Equivariant cohomology rings of toric hyperkähler manifolds. Quaternionic structures in mathematics and physics (Rome), pages 231-240 (electronic), 1999.

[15] H. Konno. Cohomology rings of toric hyperkähler manifolds. Int. J. of Math., 11(8):1001-1026, 2000.

[16] A. Knutson and T. Tao. Puzzles and (equivariant) cohomology of grassmannians. Duke Math. J., 119(2):221-260, 2003. 
[17] L. O'Shea and R. Sjamaar. Moment maps and Riemannian symmetric pairs. Math. Ann., 317(3):415-457, 2000.

[18] C. Schmid. Cohomologie équivariante des certaines variétés hamiltoniennes et de leur partie réelle. Thèse à Université de Genève, 2001.

[19] S. Tolman and J. Weitsman. On the cohomology rings of Hamiltonian T-spaces. Proc. of the Northern California Symplectic Geometry Seminar, AMS Translations Series 2, 196:251-258, 1999.

Department of Mathematics

UNIVERSITY OF TORONTO

Toronto, Ontario, M5S3G3

CANADA

E-mail address: megumi@math.toronto.edu

Department of Mathematics

University OF CONNECTICUT

Storrs, CT 06269

USA

E-mail address: tsh@math.uconn.edu

Received May 22, 2004. 